\title{
An empirical solar radiation pressure model for satellites moving in the orbit-normal mode
}

\author{
Lars Prange ${ }^{\mathrm{a}, *}$, Gerhard Beutler ${ }^{\mathrm{a}}$, Rolf Dach $^{\mathrm{a}}$, Daniel Arnold ${ }^{\mathrm{a}}$, Stefan Schaer ${ }^{\mathrm{b}}$, Adrian Jäggi ${ }^{\mathrm{a}}$ \\ ${ }^{a}$ Astronomical Institute of the University of Bern, Sidlerstrasse 5, 3012 Bern, Switzerland \\ ${ }^{b}$ Bundesamt für Landestopografie swisstopo, Seftigenstrasse 264, 3084 Wabern, Switzerland
}

\begin{abstract}
We present a family of empirical solar radiation pressure (SRP) models suited for satellites orbiting the Earth in the orbit normal (ON) mode. The proposed ECOM-TB model describes the SRP accelerations in the so-called terminator coordinate system. The choice of the coordinate system and the SRP parametrization is based on theoretical assumptions and on simulation results with a QZS-1-like box-wing model, where the SRP accelerations acting on the solar panels and on the box are assessed separately. The new SRP model takes into account that in ON-mode the incident angle of the solar radiation on the solar panels is not constant like in the yaw-steering (YS) attitude mode. It depends on the elevation angle of the Sun above the satellite's orbital plane. The resulting SRP vector acts, therefore, not only in the Sun-satellite direction, but has also a component normal to it. Both components are changing as a function of the incident angle. ECOM-TB has been used for precise orbit determination (POD) for QZS-1 and BeiDou2 (BDS2) satellites in medium (MEO) and inclined geosynchroneous Earth orbits (IGSO) based on IGS MGEX data from 2014 and 2015. The resulting orbits have been validated with SLR, long-arc orbit fits, orbit misclosures, and by the satellite clock corrections based on the orbits. The validation results confirm that—compared to ECOM2-ECOM-TB significantly (factor 3-4) improves the POD of QZS-1 in ON-mode for orbits with different arc lengths (one, three, and five days). Moderate orbit improvements are achieved for BDS2 MEO satellites-especially if ECOM-TB is supported by pseudo-stochastic pulses (the model is then called ECOM-TBP). For BDS2 IGSOs, ECOM-TB with its 9 SRP parameters appears to be over-parameterized. For use with BDS2 IGSO spacecraft we therefore developed a minimized model version called ECOM-TBMP, which is based on the same axis decomposition as ECOM-TB, but has only 2 SRP parameters and is supported by pseudo-stochastic parameters, as well. This model shows a similar performance as ECOM-TB with short arcs, but an improved performance with (3-day) long-arcs. The new SRP models have been activated in CODE's IGS MGEX solution in Summer 2018. Like the other ECOM models the ECOM-TB derivatives might be used together with an a priori model.
\end{abstract}

Keywords: satellite, precise orbit determination, solar radiation pressure, empirical model, orbit normal attitude

\section{Motivation and Previous Work}

The Center for Orbit Determination in Europe (CODE, Dach et al. 2016) has a long tradition of empirical orbit modeling. Beutler et al. (1994) introduced the Empirical Code Orbit Model (ECOM). As yaw-steering (YS) was the only relevant satellite steering for GNSS satellites in 1994, the ECOM naturally assumed that all GNSS satellites moved under the YS-attitude mode. The ECOM-decomposition was shown to be superior to the model based on the classical $\left(\vec{e}_{R}, \vec{e}_{S}, \vec{e}_{W}\right)$ decomposition proposed by Colombo (1989), where the three unit vectors represent the radial, along-track, and out-of-plane directions.

While the ECOM was initially meant to act on top of an a priori model, it turned out to be effective also as stand-alone model with a reduced set of only 5 parameters (Springer 1999) and was applied as such by CODE and other analysis centers (AC) of the International GNSS Service (IGS, Johnston et al. 2017).

\footnotetext{
${ }^{*}$ Corresponding author

Email address: lars.prange@aiub.unibe.ch (Lars Prange)
}

Problems developing with the deployment of more GLONASS satellites with their elongated spacecraft bodies could be taken into account by the ECOM2 (Arnold et al. 2015), which proved to be also effective for Galileo and QZSS spacecraft with YS attitude (Prange et al. 2017). ECOM2, as its predecessor ECOM, may act on top of an a priori model or it may be used without such a model. Like ECOM, it has been developed specifically for the YS attitude mode as it utilizes the same axis decomposition.

Prange et al. (2017) and others have, however, shown that this specialization renders those "classical" ECOM-type models unsuitable for satellites moving in the orbit normal (ON) mode. Lou et al. (2014) identified as the cause, that the SRP force component perpendicular to the solar panel introduced by reflected and diffusely scattered light photons cannot be fully covered by the ECOM during the ON-mode. They applied the ECOM with 5 parameters, but re-defined ECOM axes for POD of BeiDou2 (BDS2) satellites in the geostationary Earth orbit (GEO). Other groups partly compensate the shortcomings of the ECOM by adding constrained empirical accelerations in the along-track direction-achieving noticeable orbit improvements (e. g., Zhao et al. 2013; Guo et al. 2017). However, this 
approach is not very "physical". Montenbruck et al. (2017a) and Zhao et al. (2018) derived box-wing models for QZS-1 from empirical parameters. A more analytic approach is proposed by Darugna et al. (2018), who computed a ray-tracing model of QZS-1, based on recently published meta-data. While these models proved to be effective, they may be outdated once the spacecraft are aging and their properties do change-a phenomenon observed for GLONASS by Prange et al. (2017) and analyzed in depth by Dach et al. (2019). In general, analytic models heavily depend on up-to-date knowledge about the spacecraft. As this knowledge is usually not publicly available with a sufficient level of detail, the mentioned (semi-) analytical models are typically used in connection with an empirical model-usually a 5-parameter ECOM.

Here, we develop an ECOM that may be used for satellites in ON-mode without depending on an a priori SRP model. Such a model has the advantage that it may, in theory, be used for many different satellites in ON-mode-even for GEOs or satellites with barely known properties. When used together with an a priori SRP model, the new model is supposed to be able to absorb the residual signal caused by the reflected solar radiation during the $\mathrm{ON}$-mode more effectively than the existing ECOMs. Attempts to modify the ECOM2 by additional parameters were not entirely successful. It turned out to be more effective to use a different decomposition for the empirical accelerations, which will be introduced in Sec. 2. In Sec. 3 we discuss the differences between the YS- and ON-modes and the implications on satellite SRP modelling. In Sec. 4 we define the new ECOM based on theoretical assumptions and on a simulation study. This model is used for POD of QZS-1 and several BDS2 satellites based on MGEX data and is validated in Sec. 5. Section 6 compares the resulting orbits with MGEX products from other MGEX ACs. The results are summarized in Sec. 7.

\section{Coordinate systems for modeling SRP}

In order to define the axes of the satellite-fixed coordinate frame we need the following auxiliary vectors:

$$
\begin{array}{ll}
\vec{r} & \text { geocentric position vector of satellite } \\
\vec{r}_{S} & \text { geocentric position vector of Sun } \\
\vec{v} & \text { velocity vector of geocentric satellite motion } \\
\vec{e}_{R}= & \frac{\vec{r}}{|\vec{r}|} \\
\vec{e}_{v}= & \frac{\vec{v}}{\mid \vec{V}} \\
\vec{e}_{D}=\frac{\vec{r}_{S}-\vec{r}}{\mid \overrightarrow{\vec{r}_{S}}-\overrightarrow{\vec{r}}} \\
\vec{e}_{W}=\frac{\overrightarrow{e_{R}} \vec{e}_{v}}{\left|\vec{e}_{R} \times \vec{e}_{v}\right|},
\end{array}
$$

where $\vec{e}_{W}$ is the unit vector normal to the osculating orbital plane. The $Z$-axis of the satellite-fixed coordinate system, represented by the unit vector $\vec{e}_{Z}$, should always point to the Earth, hence

$$
\vec{e}_{Z}=-\vec{e}_{R}
$$

The $Y$-axis is oriented in different ways for satellites moving in the YS- and ON-modes:

$$
\vec{e}_{Y}=\left\{\begin{array}{ll}
-\vec{e}_{R} \times \vec{e}_{D} & \text { YS-mode } \\
\vec{e}_{W} & \text { ON-mode }
\end{array} \quad\right. \text { Fig. 1) }
$$

The unit vector associated with the coordinate axis $\vec{e}_{X}$ of the satellite-fixed coordinate system completes the right-hand system $\left(\vec{e}_{X}, \vec{e}_{Y}, \vec{e}_{Z}\right)$ and may in both cases be expressed as:

$$
\vec{e}_{X}=\vec{e}_{Y} \times \vec{e}_{Z}
$$

Equations $(2,3,4)$ define the nominal orientation of a spacecraft for both, the YS- and the ON-attitude steering. The coordinate decomposition $\left(\vec{e}_{E_{1}}, \vec{e}_{E_{2}}, \vec{e}_{E_{3}}\right)$ underlying the "classical" ECOM and ECOM2 SRP models is defined by:

$$
\begin{aligned}
& \vec{e}_{E_{3}}=\vec{e}_{D} \\
& \vec{e}_{E_{2}}=\vec{e}_{R} \times \vec{e}_{D} \\
& \vec{e}_{E_{1}}=\vec{e}_{D} \times \vec{e}_{E_{2}}
\end{aligned}
$$

For satellite motion under ON-mode one still may use the decomposition (5), where the satellite-fixed $Y$-axis is defined according to Eq. (3) for ON-motion. In this case, however, the $Y$-axis no longer coincides with the rotating $\vec{e}_{E_{2}}$ axis. Systems maintaining such a consistency of one coordinate axis with the satellite's $Y$-axis in ON-mode are e. g., the so-called RSW system, which is often used in perturbation theory (Beutler 2005, Vol. I, Chap. 6.3.5), or the orbital-plane fixed coordinate system used by Montenbruck et al. (2017a).

Here we introduce the terminator system (TERM) defined by the unit vectors $\vec{e}_{T_{1}}, \vec{e}_{T_{2}}$, and $\vec{e}_{T_{3}}$, with the Sun assumed to lie on the positive $\vec{e}_{T_{3}}$-axis. The plane spanned by $\vec{e}_{T_{1}}$ and $\vec{e}_{T_{2}}$ is thus by design the terminator plane, representing the light-shadow boundary on a spherical Earth. The $\vec{e}_{T_{1}}$-axis represents the direction to the ascending node of the satellite orbit in the terminator system. The axes of this coordinate system are defined by:

$$
\begin{aligned}
& \vec{e}_{T_{1}}=\vec{e}_{D} \times \vec{e}_{W} \\
& \vec{e}_{T_{2}}=\vec{e}_{T} \mid \\
& \vec{e}_{T_{3}}=\vec{e}_{T_{1}}
\end{aligned}
$$

The terminator system (Eq. 6) is thus unambiguously defined by the two unit vectors $\vec{e}_{D}$ and $\vec{e}_{W}$, defined by Eqs. (1). The third coordinate axis is identical in the ECOM and TERM frames, i. e., $\vec{e}_{T_{3}}=\vec{e}_{E_{3}}$. The fundamental plane of the two coordinate systems, represented by the unit vectors along the first two coordinate axes of the two systems, is therefore identical, as well. The TERM frame may be equivalent to the frame introduced by Lou et al. (2014) in order to improve the SRP model for BDS2 GEO satellites. The definition of the "virtual axis Yv" in Lou et al. (2014) is, however, not sufficiently detailed to undoubtedly conclude the equivalence with the TERM system.

\section{ON vs. YS attitude modes}

Yaw-steering has the disadvantage that rapid "noon" and "midnight" turns occur when the Sun is close to the satellite's orbital plane, i.e., when the elevation angle $\beta$ of the Sun above/below the satellite's orbital plane is small. For $\beta=0^{\circ}$, i. e., when the Sun is in the satellite's orbital plane, the satellite would theoretically have to rotate instantaneously by $\pm 180^{\circ}$ at orbit "noon" and "midnight", respectively. In order to avoid these rapid turns, satellites of some navigation (augumentation) 

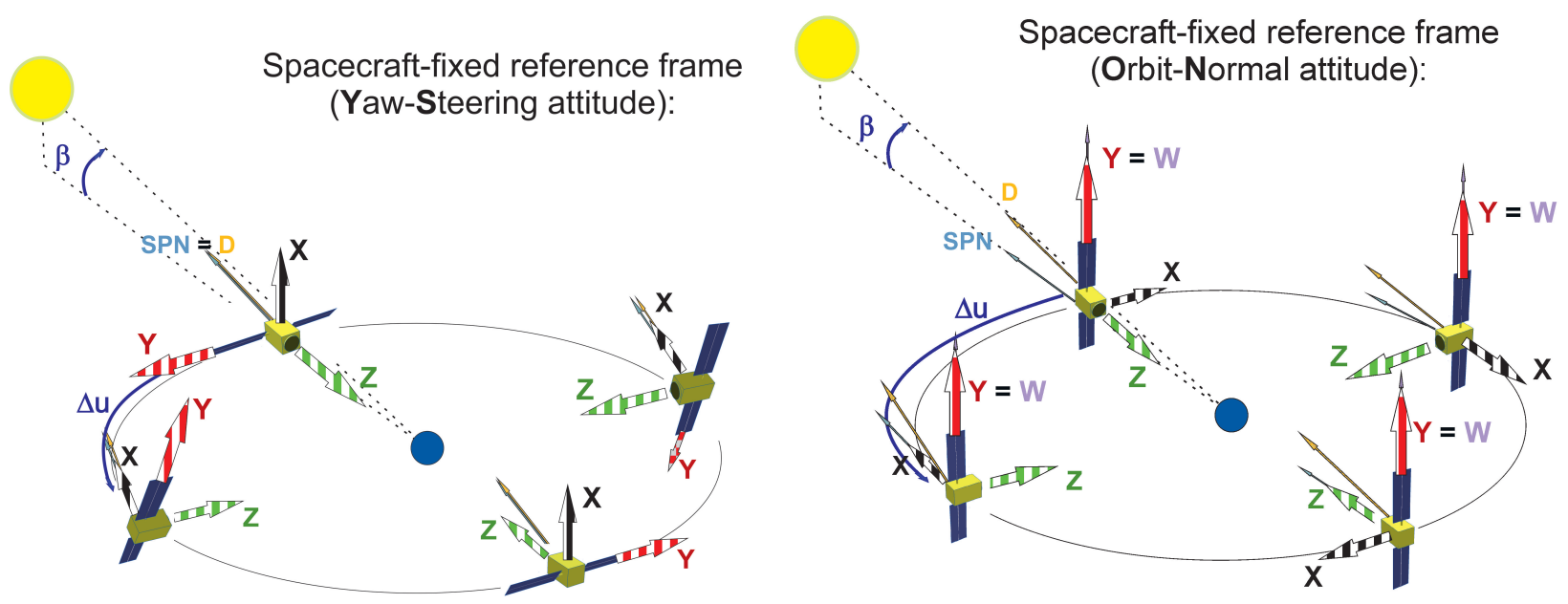

Figure 1: Satellite-fixed reference frame axes (denoted by X, Y, Z) with different attitude modes. Left: Yaw-steering (YS). Right: Orbit-normal (ON) mode. D and SPN denote the spacecraft to Sun vector and the normal of the solar panels, respectively.
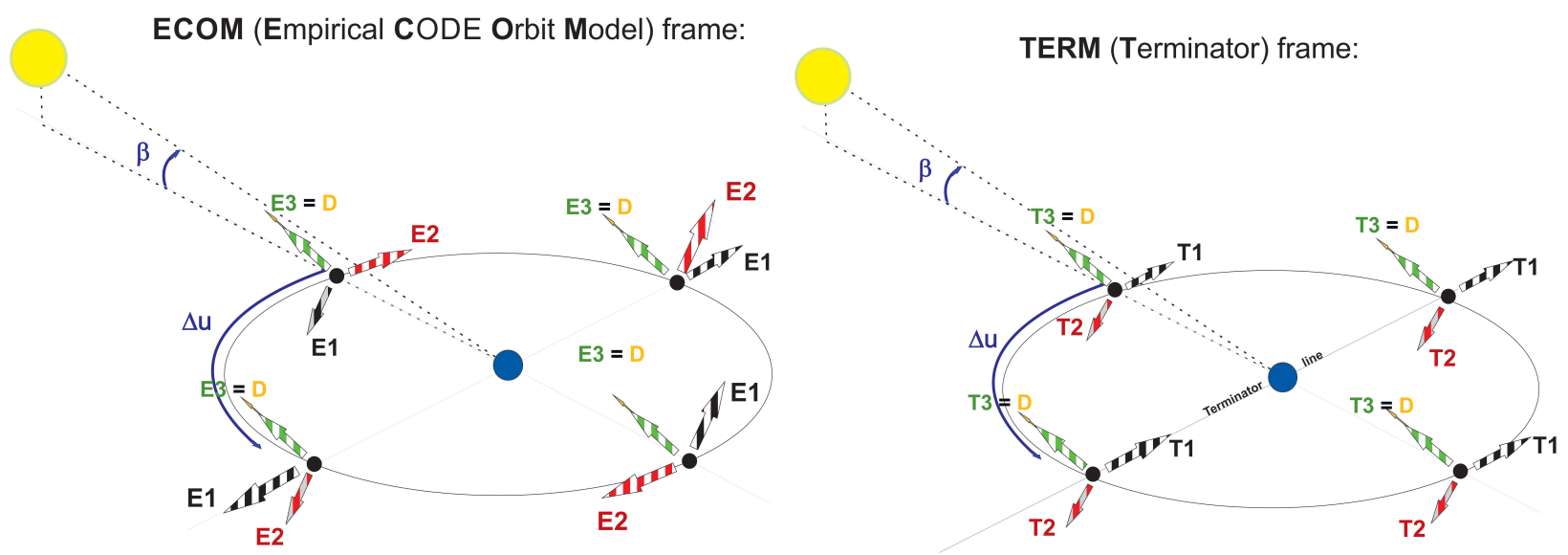

Figure 2: Coordinate frames and angles relevant for SRP modeling. Left: ECOM decomposition. Right: TERM system.

Table 1: Navigation satellites with ON-mode (Montenbruck et al. 2015).

\begin{tabular}{llll} 
System & Orbit & SVN & $\beta$-switch \\
\hline QZSS & IGSO & J001 & $\beta \approx \pm 20^{\circ}$ \\
& GEO & J003 & always \\
BDS2 & IGSO & C007, C008, & $\beta \approx \pm 4^{\circ}$ \\
& & C009, C010 & $\beta \approx \pm 4^{\circ}$ \\
& & C005 (until 2016) & $\beta \approx \pm 4^{\circ}$ \\
& MEO & C012, C013, C014 & $\beta \approx \pm 4^{\circ}$ \\
& & C015 (until ... & $\beta \approx \pm 4^{\circ}$ \\
& & $\ldots$ spring 2017) & \\
& GEO & C003, C004, C006, & always \\
BDS3 & GEO & C011, C016, C018 & always \\
SBAS & GEO & several & always \\
\hline
\end{tabular}

systems activate the ON attitude permanently (GEO satellites) or temporarily (MEO or IGSO), i. e., when $|\beta|$ drops below a certain limit (details in Tab. 1).

Under ideal YS the solar panels are always parallel to the terminator plane and normal to $\vec{e}_{D}$. The SRP on the solar panels is therefore constant. SRP variations in the $\vec{e}_{D}$ component are caused only by the satellite body, which is illuminated on the $+X,+Z$, and $-Z$ sides and is rotating w.r.t. the Sun (Arnold et al. 2015). The $Y$ surfaces are never illuminated in the YSmode.

In the $\mathrm{ON}$-mode the solar panels are not parallel to the terminator plane, except for $\beta=0^{\circ}$. They are inclined by the angle $\beta$ w.r.t. this plane. Hence, the solar panel normal $\vec{e}_{S P N}$ is not pointing to the Sun, but lies in the plane defined by the unit vectors $\left(\vec{e}_{T_{2}}, \vec{e}_{T_{3}}=\vec{e}_{D}\right)$ of the TERM system. Consequently, the power generation is reduced (by the factor $\cos \beta$ when compared to the YS-mode). Any alignment of $\vec{e}_{S P N}$ outside this plane would result in an even smaller cross section of the solar panels exposed to the Sun and thus imply a further reduced power generation. If the solar panels are nominally aligned, the incident angle of the sunlight on the solar panels is $\beta$, resulting in a SRP vector in the same plane $\left(\vec{e}_{T_{2}}, \vec{e}_{T_{3}}\right)$, which is constant for a constant $\beta$. As opposed to the YS-mode one of the $Y$ surfaces is always illuminated (except for $\beta=0^{\circ}$ ). The area of its cross section as seen from the Sun remains the same over the entire revolution of the satellite for a constant angle $\beta$. In 
the ON-mode the satellite body is rotating around the inertial $\vec{e}_{Y}=\vec{e}_{W}$-axis. This results in a periodic illumination of the $\pm X$ and $\pm Z$ surfaces.

\section{SRP parametrization under YS- and ON-modes}

The ECOM SRP models decompose the perturbing accelerations as a linear combination of the three orthogonal unit vectors $\vec{e}_{E_{1}}, \vec{e}_{E_{2}}, \vec{e}_{E_{3}}$ defined by Eqns. (5):

$$
\vec{a}=\vec{a}_{0}+E 3(\Delta u) \vec{e}_{E_{3}}+E 2(\Delta u) \vec{e}_{E_{2}}+E 1(\Delta u) \vec{e}_{E_{1}},
$$

where $\vec{a}_{0}$ is an optional and freely selectable a priori model, $\Delta u=u-u_{s}$ is the difference of the arguments of latitude of the satellite $u$ and the Sun $u_{s}$. Arnold et al. (2015) write the components of ECOM2 as truncated Fourier series using the angular argument $\Delta u$ :

$$
\begin{aligned}
& E 3(\Delta u)=\mathbf{E 3 0}+\mathbf{E 3 C 2 u} \cos 2 \Delta u+\mathbf{E 3 S} 2 \mathbf{u} \sin 2 \Delta u \\
& E 2(\Delta u)=\mathbf{E} 20+\mathbf{E} \mathbf{E} \cos 4 \Delta u+\mathbf{u} \sin 4 \Delta u \\
& E 1(\Delta u)=\mathbf{E 1 0}+\mathbf{E} 1 \mathbf{C} 1 \mathbf{u} \cos \Delta u+\mathbf{E} 1 \mathbf{S} 1 \mathbf{u} \sin \Delta u
\end{aligned}
$$

$E 3(\Delta u)$ only has even-order, $E 1(\Delta u)$ only odd-order harmonics of the orbital period. In the YS-mode SRP caused by the solar panels is captured uniquely by the coefficient E30, while the other coefficients are solely needed to model SRP caused by the satellite body.

In order to accommodate satellites in the ON-mode, as well, one might retain the decomposition Eq. (7) and replace the definition of $\vec{e}_{E_{2}}$ according to Eq. (3) for the ON-mode. The resulting decomposition Eq. (7) would not be orthogonal in the ON-mode, however. Alternatively, additional parameters could be added to the ECOM2. When assuming a simplified satellite consisting only of solar panels and of a spherical body moving in the $\mathrm{ON}$-mode, one obtains a constant component along $\vec{e}_{E_{3}}$ for a constant angle $\beta$ and components with a once-perrevolution (1pr) signature along the vectors $\vec{e}_{E_{2}}$ and $\vec{e}_{E_{1}}$. As the rotation of the $\vec{e}_{E_{2}}$ - and $\vec{e}_{E_{1}}$-axes parallel to the terminator in the ECOM coordinate system is highly non-uniform for small $\beta$-angles, the spectral decomposition of SRP would consist of many harmonics of the revolution period.

Based on this insight an alternative decomposition of the SRP accelerations with an alternative parametrization seems appropriate. One such coordinate system is the TERM system defined by Eq. (6). In analogy to Eq. (7) and considering a slowly changing $\beta$-angle the perturbing accelerations may then be described as:

$$
\vec{a}=\vec{a}_{0}+T 1(\Delta u, \beta) \vec{e}_{T_{1}}+T 2(\Delta u, \beta) \vec{e}_{T_{2}}+T 3(\Delta u, \beta) \vec{e}_{T_{3}}
$$

Unlike the ECOM, the TERM decomposition is not rotating with the satellite in inertial space. On the other hand, the new tripod is not entirely fixed in inertial space, because both, the Sun and the orbit normal vector $\vec{e}_{W}$ defining the TERM system are not fixed in inertial space, either.

\subsection{SRP caused by the solar panels in the ON-mode}

Solar radiation falling on the solar panels is either absorbed (about 75\%), specularly (about 20\%) or diffusely reflected (about 5\%) by the panels (Rodriguez-Solano et al. 2012). Assuming that the entire solar radiation is absorbed, the following parametrization would represent SRP due to the solar panels in the ON-mode:

$$
\begin{aligned}
T 1 & =0 \\
T 2 & =0 \\
T 3 & =-\cos \beta D_{a},
\end{aligned}
$$

where $D_{a}$ is equivalent to that part of E30 in Eq. (8) caused by the radiation absorbed by the solar panels. A better approximation results, if we take absorption and specular reflection into account. Acceleration due to specular reflection acts along the surface normal vector $\vec{e}_{S P N}=\cos \beta \vec{e}_{T_{3}}+\sin \beta \vec{e}_{T_{2}}$ to the solar panels (Milani et al. 1987) and therefore may be calculated as:

$$
\begin{aligned}
\vec{a}_{s} & =-2 D_{s} \cos \beta \vec{e}_{S P N} \\
& =-2 D_{s} \cos \beta\left(\cos \beta \vec{e}_{T_{3}}+\sin \beta \vec{e}_{T_{2}}\right) \\
& =-D_{s}\left(2 \cos ^{2} \beta \vec{e}_{T_{3}}+\sin 2 \beta \vec{e}_{T_{2}}\right)
\end{aligned}
$$

where $2 D_{s}$ is the momentum (per mass) gained by a satellite due to specular reflection with its solar panels exposed normal to vector $\vec{e}_{D}$. By combining the effects due to absorbtion and specular reflection one obtains:

$$
\begin{aligned}
T_{1} & =0 \\
T_{2} & =-D_{s} \sin 2 \beta \\
T_{3} & =-\cos \beta\left(D_{a}+2 D_{s} \cos \beta\right) \\
& \approx-\cos \beta\left(D_{a}+2 D_{s}\right) \\
& =-\cos \beta D_{0},
\end{aligned}
$$

where $D_{a}+2 D_{s} \doteq D_{0}=$ E30 is equivalent to the acceleration gained by a corresponding satellite in the YS-mode due to absorption and specular reflection. The approximation in Eqs. (12) holds for small angles $\beta$. Equations (12) tell that SRP due to the solar panels for satellites in ON-mode can be taken into account by a 2-parameter model, namely by the parameters $D_{0}=D_{a}+2 D_{s}$ and $D_{s}$. The acceleration in the $\vec{e}_{T_{2}}$ direction is solely caused by the reflected fraction of the solar radiation, while the $\vec{e}_{T_{3}}$ component is caused by both-absorption and reflection. Note that-as opposed to the YS - the SRP parameters depend on the $\beta$ angle in the ON-mode. As the orbital planes of GNSS satellites are typically precessing w. r. t. the Sun, their $\beta$ angles are slowly changing in time (typically by the order of about $1 \%$ d).

\subsection{SRP simulation for the $O N$-mode}

The assumptions made in Sect. 4.1 are confirmed and the more complex SRP accelerations due to the spacecraft body are assessed with a simulation study for a QZS-1-like spacecraft. The satellite mass, size, and optical surface properties are taken from Cabinet Office (2017) and Montenbruck et al. (2017a). For the sake of simplicity we assume a circular orbit with the semi major axis corresponding to a GEO. The acceleration caused by SRP is computed separately for each illuminated spacecraft surface according to Milani et al. (1987). Earth shadow transitions are taken into account. 

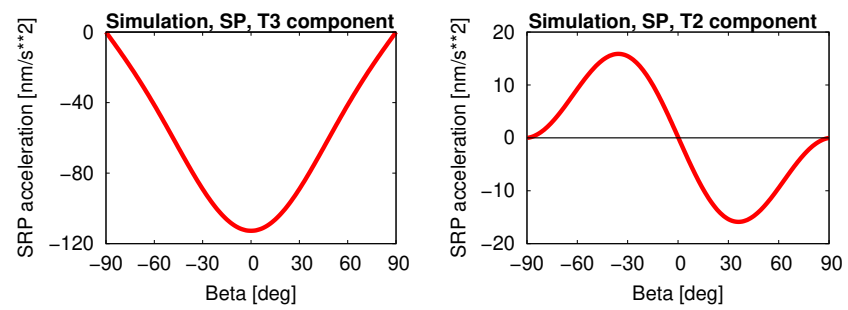

Figure 3: Simulated SRP due to solar panels (SP) projected into the TERM system as a function of $\beta\left(-90^{\circ}<\beta<+90^{\circ}\right)$. Left: T3 component. Right: T2 component.

In a first step we simulate the SRP caused by the solar panels. The simulation results in Fig. 3 confirm the considerations from Sect. 4.1: The SRP due to the solar panels splits up into a component in $T_{3}$ and a component with a smaller amplitude in $T_{2}$. Both components are constant with respect to $\Delta u$, but depend on $\cos \beta$ and $\sin 2 \beta$, respectively.

When taking into account the spacecraft body we obtain a more complex SRP signal-as outlined in Sect. 3. Significant SRP accelerations occur in all three components of the TERM system and they all depend on $\Delta u$ and $\beta$ (Fig. 4). By adding the contributions from solar panels and satellite body we obtain the simulated total SRP acceleration. In analogy to Eq. (8) the resulting accelerations can be approximated by a truncated Fourier series using the angular argument $\Delta u$. Selecting the most significant Fourier terms (in our case those with amplitudes $>1 \mathrm{~nm} / \mathrm{s}^{2}$ ), we obtain the following parameterization for a candidate ECOM-T (i. e., an ECOM SRP model, whose axes are defined by the TERM reference frame, Eq. 6):

$$
\begin{aligned}
& T 3(\Delta u)=\mathbf{T 3 0}+\mathbf{T} 3 \mathbf{C} 2 \mathbf{u} \cos 2 \Delta u+\mathbf{T} 3 \mathbf{S} 2 \mathbf{u} \sin 2 \Delta u \\
&+\mathbf{T 3 C 4} \cos 4 \Delta u+\mathbf{T} 3 \mathbf{S} 4 \mathbf{u} \sin 4 \Delta u \\
& T 2(\Delta u)=\mathbf{T 2 0}+\mathbf{T} \mathbf{C} 2 \mathbf{u} \cos 2 \Delta u+\mathbf{T} 2 \mathbf{S} 2 \mathbf{u} \sin 2 \Delta u \\
& T 1(\Delta u)=
\end{aligned}
$$

Note, that our T20- and T30-coefficient designations have nothing to do with the ROCK radiation pressure models (Fliegel et al. 1992) of the GPS system. Figure 5 shows that all coefficients of the ECOM-T depend on the angle $\beta$. The angle $\beta$ is changing slowly in time (by about $1^{\circ} / \mathrm{d}$ ) for typical GNSS orbits. As a consequence, the SRP coefficients in Eq. (13) are time-dependent, as well. This may be problematic when estimating long-arcs, e.g., over three days. Therefore, we express each SRP coefficient in Eq. (13) as a function of $\beta$, fit it with a Fourier series and retain the most significant term. Hereby we focus on $\beta$-angles within $\pm 30^{\circ}$. The coefficients of the resulting ECOM-TB SRP model (i. e., an ECOM-T, whose terms are functions of the $\beta$-angle) are much less variable in time:

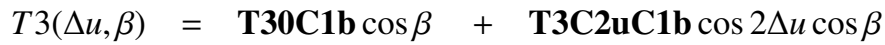

$$
\begin{aligned}
& + \text { T3S2uC1b } \sin 2 \Delta u \cos \beta \\
& + \text { T3C4uC1b } \cos 4 \Delta u \cos \beta \\
& + \text { T3S4uC1b } \sin 4 \Delta u \cos \beta \\
& T 2(\Delta u, \beta)=\mathbf{T 2 0 S 3 b} \sin 3 \beta+\mathbf{T} 2 \mathbf{C} 2 \mathbf{u S} 2 \mathbf{b} \cos 2 \Delta u \sin 2 \beta \\
& +\mathbf{T} 2 \mathbf{S} 2 \mathbf{U S 2} 2 \mathbf{b} \sin 2 \Delta u \sin 2 \beta \\
& T 1(\Delta u, \beta)= \\
& \text { T1S2uC1b } \sin 2 \Delta u \cos \beta
\end{aligned}
$$

Apart from the "full" ECOM-TB (Eq. 14) we also use modified versions of this model:

$$
\begin{aligned}
& T 3(\Delta u, \beta)=\mathbf{T 3 0 C} \mathbf{1 b} \cos \beta \\
& T 2(\Delta u, \beta)=\mathbf{T 2 0 S} 2 \mathbf{b} \sin 2 \beta \\
& T 1(\Delta u, \beta)=0
\end{aligned}
$$

The "minimized" ECOM-TBM (Eq. 15) is reduced to the two SRP parameters needed to describe the SRP due to the solar panels (see derivation in Eq. 12). It is designed to serve as a supplement for an a priori model that describes the SRP due to the satellite body. When used without an a priori model, the SRP contribution from the spacecraft body might instead be absorbed by pseudo-stochastic parameters (Beutler et al. 1994), e.g., by pulses set up every $12 \mathrm{~h}$ in radial, along-, and cross-track directions-resulting in the ECOM-TBMP. The ECOM-TB versions used in this work are summarized in Tab. 2.

\begin{tabular}{lccl} 
Model & Table 2: Versions of the ECOM-TB SRP model. \\
\#Parm. & Definition & Pulses \\
\hline ECOM-TB & 9 & see Eq. 14 & none \\
ECOM-TBP & 9 & see Eq. 14 & every $12 \mathrm{~h}$ \\
ECOM-TBM & 2 & see Eq. 15 & none \\
ECOM-TBMP & 2 & see Eq. 15 & every $12 \mathrm{~h}$ \\
\hline
\end{tabular}

\section{Confrontation with real data}

We now use the SRP models defined by Eqs. (13) and (14) in a POD and clock determination based on IGS MGEX data. Our analysis focuses on the following time intervals: In interval one (I1: DOY 357/2014 to 108/2015) QZS-1 and all BDS2 MEO and BDS2 IGSO satellites enter the ON-mode at some point in time. This includes also the BDS2 satellites SVN C008, C010 (both IGSO), and C012 (MEO), which are tracked by the International Laser Ranging Service (ILRS, Pearlman et al. 2002). At some point in time in interval two (I2: DOY 230 to 294/2015) QZS-1 and the BDS2 satellites SVN C005, C009 (both IGSO), and C015 (MEO) enter the ON-mode. Time interval three (I3: DOY 230 to 250/2015) is a sub-interval of I2. The processing strategy, data base, background models, and validation methods have been described in detail by Prange et al. (2017). They agree with the COM product published for the aforementioned time periods. GEO satellites are not part of the COM product. Therefore, they are not considered in this study, as well. SRP is not the only issue limiting the POD of GEO satellites. Observation geometry, continuous ambiguity handling, orbit arc length, maneuver handling and other aspects are challenging, as well. These issues deserve further research, which is, however, beyond the scope of this study.

\subsection{SRP coefficients of $Q Z S-1$}

QZS-1 is selected for testing the ECOM-TB for the following reasons: The satellite enters the ON-mode at the sizeable $\beta$-angles of $\pm 20^{\circ}$ and stays in this mode for a long time (about 45 days). Therefore, temporal changes of SRP parameters can 

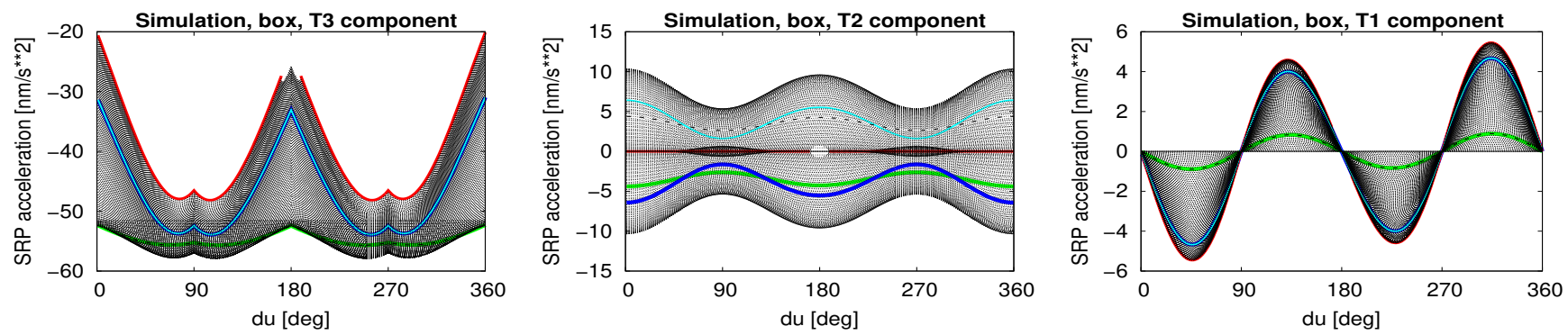

Figure 4: Simulated SRP due to spacecraft box in the TERM system as a function of $\Delta u$ for a range of $\beta$-angles $\pm 90^{\circ}$. Colors represent following $\beta$-angles: green: $-80^{\circ}$, blue: $-30^{\circ}$, red: $0^{\circ}$, cyan: $+30^{\circ}$, gray, dotted: $+80^{\circ}$. Left: T3 component. Center: T2 component. Right: T1 component.
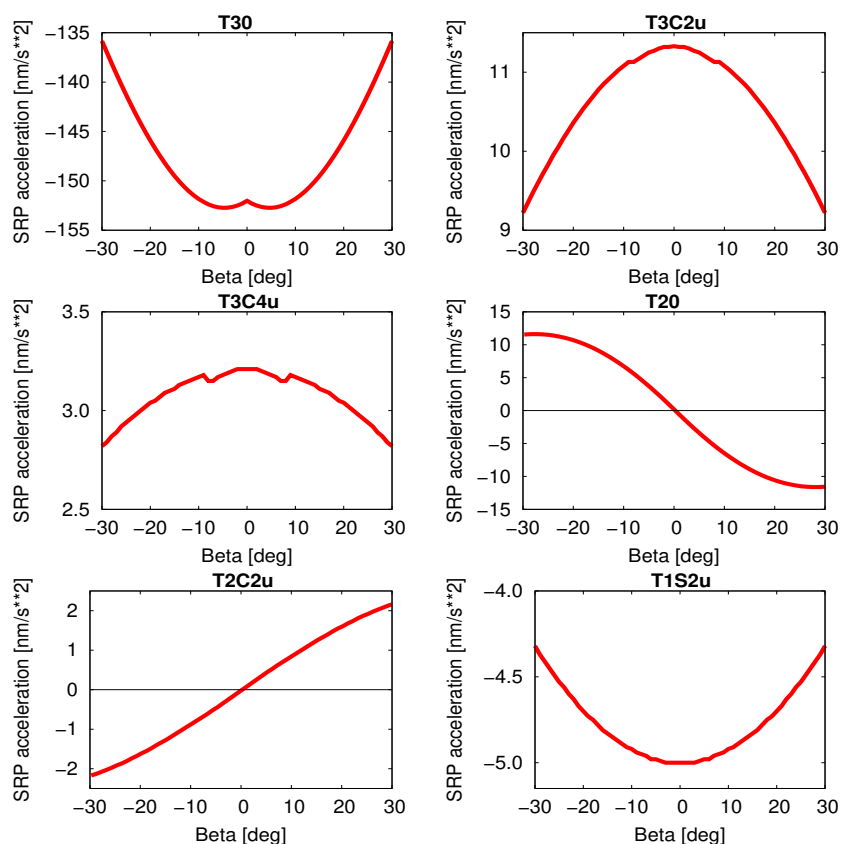

Figure 5: Significant coefficients of the proposed ECOM-T SRP model as a function of the $\beta$-angle $\left(\beta\right.$-range $\left.\pm 30^{\circ}\right)$.

be studied and compared with the expectations from the simulation. Due to the large $\beta$-angle at transition, the effect of YSON-YS attitude mode changes is very pronounced, simplifying the detection of mode transitions. The properties of the satellite are relatively well known. The spacecraft is well tracked by the IGS MGEX stations in Asia, Australia, and the Pacific regiona prerequisite for POD and precise clock determination. QZS-1 is also observed by the ILRS-permitting SLR orbit validation. Estimates of the stable satellite clock might be used for orbit validation, as well. Therefore, we focus on QZS-1 and data from I3 in our initial analysis.

Figure 6 compares the simulated T30 and T20 coefficients of ECOM-T (Eq. 13) with the corresponding parameters estimated from real data. The estimated T30 coefficient (Fig. 6, left) differs from the simulated one only by about $2 \mathrm{~nm} / \mathrm{s}^{2}$ and shows the same dependency on $\cos \beta$. It might, therefore, be represented by $\mathrm{T} 30 \mathrm{C} 1 \mathrm{~b}$ (in the example with a value of about $-153 \mathrm{~nm} / \mathrm{s}^{2}$ ) as emerging from Eq. 14.

The estimated T20 coefficient (Fig. 6, right) is larger than
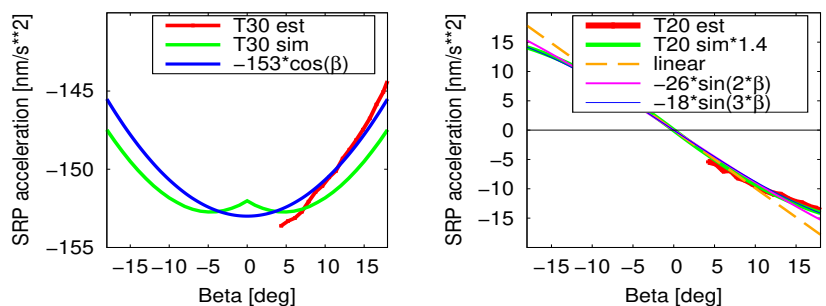

Figure 6: Comparison of estimated ("est", within time interval I3) and simulated ("sim") coefficients T20 and T30 of the ECOM-T SRP model for QZS-1 with approximation functions. Left: T30. Right: T20.

the simulated one by a factor of about 1.4-indicating that the simulation underestimates the SRP acceleration due to the solar panels. Apart from that both curves have a similar shape. The other curves show examples of $\beta$-dependent functions suited to fit T20, as well. The blue curve corresponds to the coefficient T20S3b (here with a value of about $-18 \mathrm{~nm} / \mathrm{s}^{2}$ ) as emerging from Eq. 14. All three examples $(\mathrm{T} 20 \mathrm{~b} \cdot \beta, \mathrm{T} 20 \mathrm{~S} 2 \mathrm{~b} \cdot \sin 2 \beta$, $\mathrm{T} 20 \mathrm{~S} 3 \mathrm{~b} \cdot \sin 3 \beta)$ are acceptable approximations for the curve of estimated T20 within $\beta$-angles $\pm 20^{\circ}$, which is the $\beta$-range relevant for QZS-1. A linear approximation would be sufficient for BDS2 MEO and IGSO satellites, which are in the ON-mode only for $|\beta| \leq 4^{\circ}$. As the T20-curve is flattening for larger $\beta$ angles (see Fig. 5), the linear approximation may, however, not be sufficient for GEO satellites with $\beta$-angles of up to $\pm 23.5^{\circ}$ (plus few more degrees if their inclination deviates from zero). For the ECOM-TB we therefore stay with the T20S3b coefficient as suggested by Eq. 14. In summary, Fig. 6 confirms the basic findings from Sect. 4.2 (particularly those concerning the dependency of the ECOM-T coefficients on $\beta$ ), but also shows the limitations of the simulation in predicting the actual size of the SRP coefficients.

Subsequently, we apply the ECOM-TB (Eq. 14) to MGEX data of QZS-1 from the intervals I1 and I2. Figure 7 confirms that the agreement between estimated and simulated ECOM-TB parameters of QZS-1 is usually better than $5 \mathrm{~nm} / \mathrm{s}^{2}$. The most important coefficients $\mathrm{T} 30 \mathrm{C} 1 \mathrm{~b}$ and $\mathrm{T} 20 \mathrm{~S} 3 \mathrm{~b}$ are relatively constant within large parts of the shown $\beta$-range. The coefficients related to the T2-component, however, are disturbed for $\beta$-angles close to zero. This is related to their pre-factors containing $\sin (n \cdot \beta)$, i. e., they are singular for $\beta=0$. Note that some coefficients (e. g., T30C1b, T3C2uC1b, T1S2uC1b) differ 

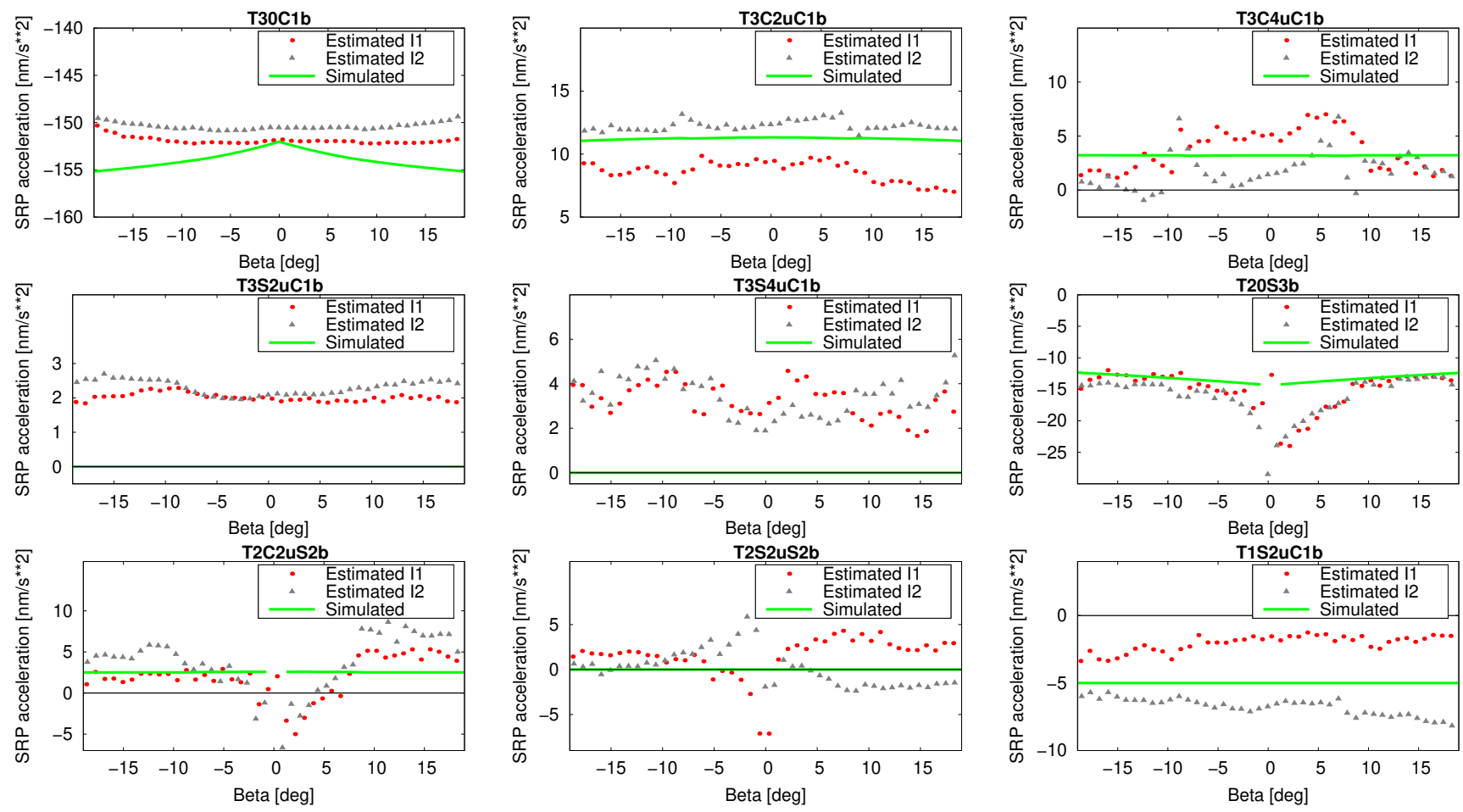

Figure 7: Estimated (within time intervals I1 and I2) and simulated coefficients of ECOM-TB SRP model for QZS-1 as a function of the $\beta$-angle.

significantly in both ON-passes, i. e., between I1 and I2. Within I1 the $\beta$-angle of QZS-1 changes from negative to positive values, within $\mathrm{I} 2$ from positive to negative. These differences might be related to the eccentricity of QZS-1's orbit, to an attitude slightly deviating from perfect $\mathrm{ON}$, to asymmetric satellite properties, or thermal emission. The estimated coefficient $\mathrm{T} 3 \mathrm{C} 4 \mathrm{uC} 1 \mathrm{~b}$ seems to be disturbed by some unexpected signal and is almost stochastic. Note that the coefficients $\mathrm{T} 3 \mathrm{~S} 2 \mathrm{uC} 1 \mathrm{~b}$ and $\mathrm{T} 3 \mathrm{~S} 4 \mathrm{uC} 1 \mathrm{~b}$, which - according to the simulation-are supposed to be zero, are significantly different from zero and show a good agreement in both intervals. It is likely that they absorb a signal, which is not considered in the simulation (e.g., thermal radiation).

In summary, most of the estimated ECOM-TB coefficients are relatively constant in time (except if they are close to singularity). Therefore the ECOM-TB is well suited for stacking orbit parameters in a long-arc POD.

\subsection{QZS-1: orbit and clock validation}

The QZS-1 orbits and satellite clock corrections generated in Sect. 5.1 are validated by SLR residuals and by the computation of the RMS of a linear fit through the epoch-wise clock corrections for each day. Apart from the attitude and the SRP model applied during ON-seasons the methods and background models are the same as those used by Prange et al. (2017). The operational COM product based on the ECOM2 SRP model is included as an additional reference. Figure 8 shows that the large SLR residuals (almost $1.5 \mathrm{~m}$ ) and RMS of linear clock fit (up to $3.5 \mathrm{~ns}$ ) QZS-1 has in the regular COM solution during ONmode, can be significantly reduced by using the ECOM-TB.
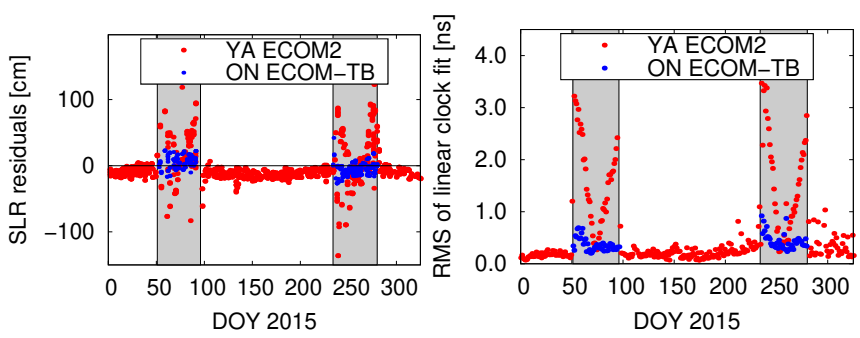

Figure 8: SLR residuals (left) and RMS of linear fit through clock corrections (right) of QZS-1 in 2015. Shaded zones: ON-mode on; Red: operational COM solution always applying YS and ECOM2 SRP model; Blue: re-processed solution with correct attitude and ECOM-TB SRP model during ON-seasons.

The scatter of the SLR residuals is almost as small as under the YS-regime. During the interval I2 the SLR offset is comparable with that in the YS-mode. In the interval I1 it is shifted. The clock RMS can be reduced to values around $0.35 \mathrm{~ns}$ during most of the ON-mode operation, which is still larger than during the YS intervals. However, in the first days of both ONintervals in 2015 the clock RMS is larger (0.5 - 1 ns) than in the remaining interval. The SLR residuals do not show comparable problems. These problems might be caused by changing thermal conditions onboard the spacecraft after the attitude change mode from YS to ON.

Figure 9 shows that the QZS-1 orbits with arc-lengths of three days are better than the corresponding 1-day solutions when ECOM-TB is applied. They can obviously benefit from the advatages of long-arc solutions, which were already pointed out by Lutz et al. (2016). The 5-day results are not as good as the 3-day results-indicating that the QZS-1 solution shown 

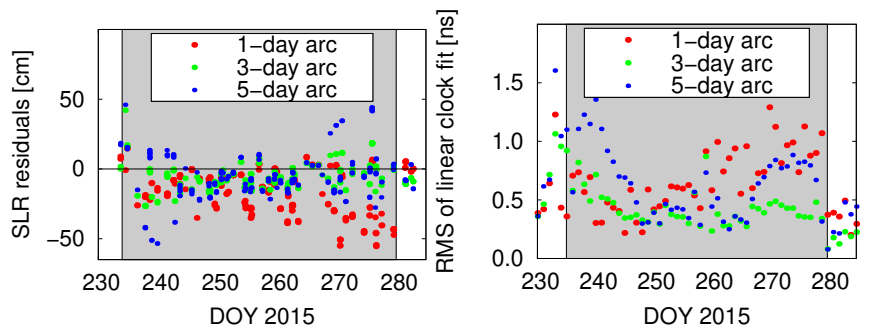

Figure 9: SLR residuals (left) and RMS of linear fit through clock corrections (right) of QZS-1 during interval I2 in 2015. Orbits are computed with ECOM-TB, but different arc-lengths. Shaded zones: ON-mode on.

here is still suffering from accumulated modelling deficiencies (e. g., missing albedo and antenna thrust modelling), which are partly absorbed by the arc-specific parameters. In summary, by replacing the ECOM2 by the ECOM-TB we are able to significantly reduce the inter quartile range (IQR) of the SLR residuals $(62.0 \rightarrow 15.2 \mathrm{~cm})$, the RMS of the linear clock fit $(1.43 \rightarrow$ $0.36 \mathrm{~ns})$, the median of the orbit misclosures $(42.4 \rightarrow 14.2 \mathrm{~cm})$, and the RMS of the long-arc orbit fit $(14.1 \rightarrow 5.6 \mathrm{~cm})$ in a COM-style 3-day long-arc orbit solution of QZS-1 during ONmode periods (Tab. 4).

\subsection{BDS2: SRP coefficients}

In analogy to QZS-1 the ECOM-TB is validated for BDS2 MEO and IGSO satellites in the time intervals I1 and I2. As opposed to QZS-1 we study all ECOM-TB versions listed in Tab. 2. Note however, that not all model versions are applied to all time windows mentioned above. Figures 10 and 11 show the T30C1b and T20S3b coefficients estimated for 1-, 3-, and 5-day arc solutions of BDS2 MEO and IGSO satellites, respectively. The better solutions are relatively constant in time (i.e., for different $\beta$-angles).

With short arcs MEO satellites with the full ECOM-TB show T30C1b coefficients, which are relatively constant in time and agree well among the different spacecraft. For long arcs the day-to-day values show a few large outliers. Introducing pulses (ECOM-TBP) helps to stabilize the solution. The solutions with ECOM-TBM and ECOM-TBMP are more homogeneous for all arc-lengths. The average value of the T30C1b coefficient is around $-130 \mathrm{~nm} / \mathrm{s}^{2}$, which is equivalent to the value of E30 of ECOM2 (in Eq. 8) under YS.

The T20S3b coefficient of ECOM-TB shows significant dayto-day differences - in particular for the MEO solutions with short arcs. Longer (especially 3-day) arcs and pulses moderately reduce these differences. The T20S3b coefficient of the ECOM-TBM solution may have extreme values-indicating that this SRP model is not sufficient. When pulses are added (ECOM-TBMP) it is, however, similar to the models ECOM-TB and ECOM-TBP.

For IGSO spacecraft analyzed with the full ECOM-TB, the T30C $1 \mathrm{~b}$ coefficient can be well determined for short arcs, only. For long arcs, however, the T30C1b values show large deviations from the values achieved with the short arc solutions. These deviations can be significantly reduced by introducing pulses (ECOM-TBP). A systematic offset remains, however.
Table 3: Validation statistics of COM solutions based on ECOM2 in the time intervals I1 and I2. (ECOM2-P stands for ECOM2 plus pulses; IQR for inter quartile range; BI for BDS2 IGSO; BM for BDS2 MEO; Q1 for QZS-1; $3 \mathrm{~d}$ for an arc-length of three days)

\begin{tabular}{lccc} 
Sat. & ECOM2 & ECOM2 & ECOM2-P \\
-typ & YS & ON & ON \\
& 3 d & 3 d & 3 d \\
\hline \multicolumn{4}{c}{ SLR residuals $[\mathrm{cm}]:$ median \pm IQR } \\
\hline BI & $-3.0 \pm 6.1$ & $1.5 \pm 20.5$ & $9.2 \pm 33.1$ \\
BM & $-1.1 \pm 4.2$ & $-3.8 \pm 21.0$ & $-2.5 \pm 17.1$ \\
Q1 & $-10.4 \pm 9.3$ & $9.4 \pm 62.0$ & $15.7 \pm 56.4$ \\
\hline \multicolumn{4}{c}{ RMS of linear clock fit [ns]: median \pm IQR } \\
\hline BI & $0.87 \pm 0.77$ & $1.72 \pm 1.32$ & $1.22 \pm 0.79$ \\
C005 & $2.49 \pm 1.43$ & $2.23 \pm 1.92$ & $1.72 \pm 1.31$ \\
BM & $0.45 \pm 0.55$ & $1.61 \pm 0.61$ & $0.69 \pm 0.75$ \\
C012 & $0.34 \pm 0.12$ & $1.52 \pm 0.57$ & $0.36 \pm 0.15$ \\
Q1 & $0.18 \pm 0.12$ & $1.43 \pm 1.47$ & $1.41 \pm 1.45$ \\
\hline \multicolumn{4}{c}{ 3d orbit misclosures [cm]: median \pm IQR } \\
\hline BI & $7.9 \pm 8.2$ & $55.9 \pm 49.0$ & $16.3 \pm 14.0$ \\
BM & $7.0 \pm 6.1$ & $29.2 \pm 58.7$ & $8.0 \pm 7.7$ \\
Q1 & $10.0 \pm 11.7$ & $42.4 \pm 59.9$ & $37.2 \pm 63.1$ \\
\hline RMS of 3-day long arc orbit fit [cm]: median \pm IQR \\
\hline BI & $1.6 \pm 1.1$ & $23.0 \pm 18.1$ & $31.9 \pm 21.3$ \\
BM & $1.2 \pm 0.6$ & $10.1 \pm 13.9$ & $8.8 \pm 5.4$ \\
Q1 & $3.6 \pm 2.2$ & $14.1 \pm 16.8$ & $17.6 \pm 18.4$ \\
\hline \multicolumn{4}{c}{}
\end{tabular}

For the models ECOM-TBM and ECOM-TBMP the estimates of $\mathrm{T} 30 \mathrm{C} 1 \mathrm{~b}$ are stable in time for all arc-lengths. The value of about $-122 \mathrm{~nm} / \mathrm{s}^{2}$ is close to the value of the ECOM 2 coefficient E30 in the YS-regime, i. e., it is realistic. The different sizes of T30C1b for MEO and IGSO spacecraft indicate larger masses or design differences of the IGSO satellites.

For IGSO short-arcs, the T20S3b coefficient is of the same quality for all ECOM-TB model versions. Its value is less time variable than for the MEO satellites. For longer arcs it is, however, degraded. When pulses are introduced (ECOM-TBP and ECOM-TBMP), the long-arc solutions are of the same quality as the short-arc solution and rather stable in time.

These results indicate that the main coefficients of ECOM-TB cannot be determined for BDS2 satellites with the same quality as for QZS-1. Especially the long-arc solutions are affected by problems to be discussed later in this analysis.

\subsection{BDS2: orbit and clock validation}

In analogy to Sect. 5.2 we validate the BDS2 solutions described in Sect. 5.3. The statistics are summarized in Tab. 4. Note, that the ECOM-TBM has been tested only in the interval I2. Therefore, no SLR residuals and no satellite clocks of SVN C012 are available for this model. For the purpose of comparison the corresponding performance figures of solutions using ECOM2 and ECOM2-P (i. e., an ECOM2 plus pulses every $12 \mathrm{~h}$ ) are listed in Tab. 3.

In the subsequent analysis we distinguish between MEO and IGSO satellites on the one hand and between methods for absolute and relative orbit validation on the other hand. As "absolute" we consider methods, characterizing how well 

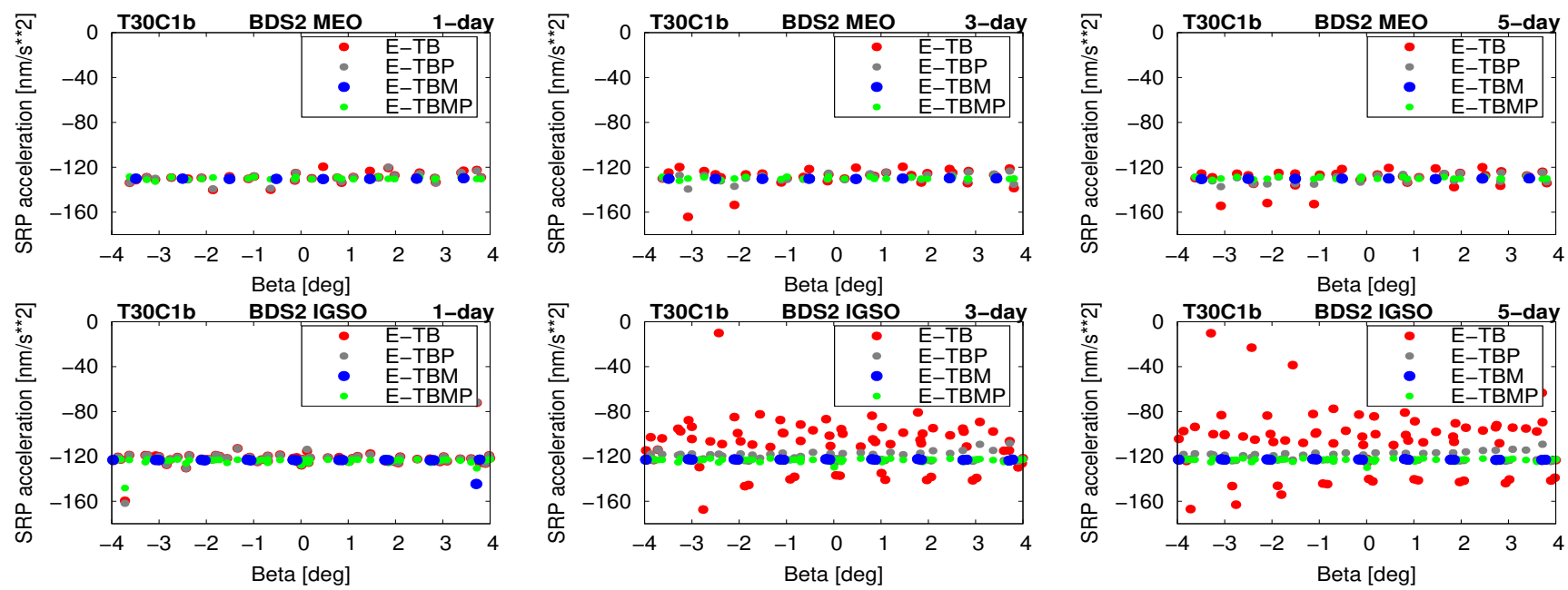

Figure 10: T30C1b SRP coefficient as a function of the $\beta$-angle for different versions of ECOM-TB models. ECOM is abbreviated as "E". Top: BDS2 MEO satellites. Bottom: BDS2 IGSO satellites. Left column: 1-day orbit arcs. Middle column: 3-day orbit arcs. Right column: 5-day orbit arcs.
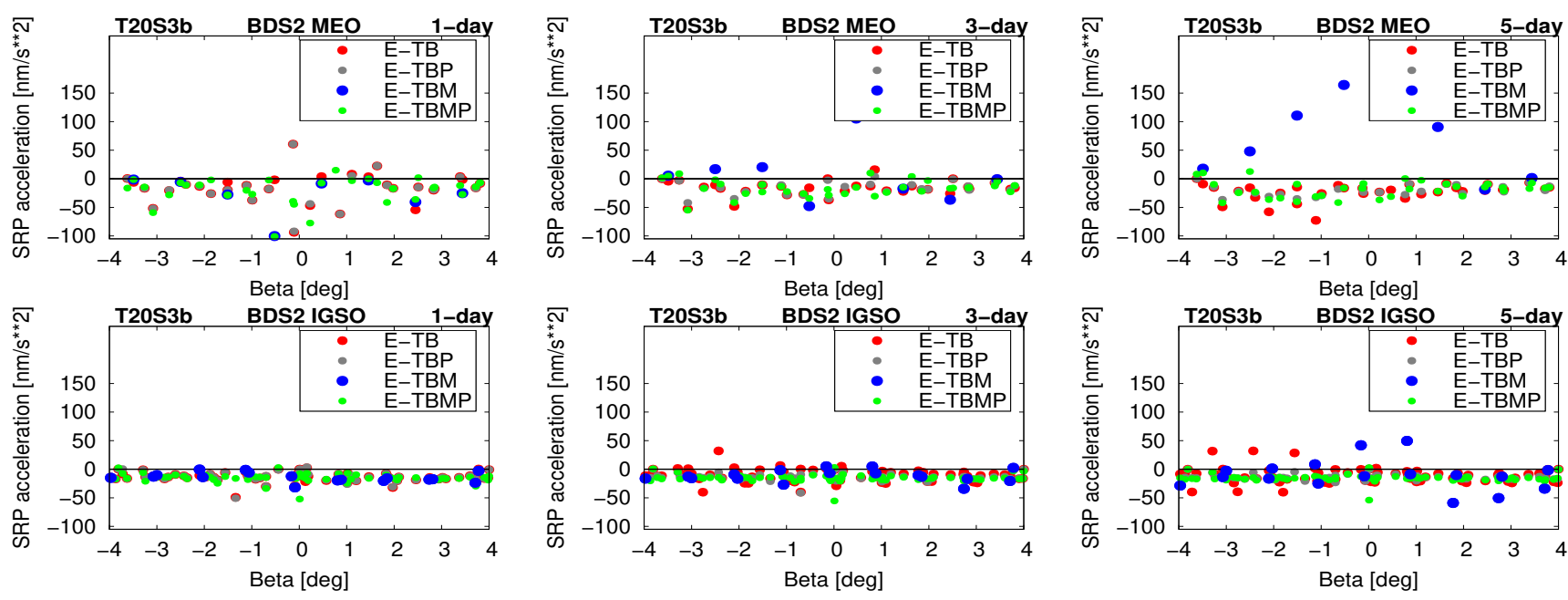

Figure 11: T20S3b SRP coefficient as a function of the $\beta$-angle for different versions of ECOM-TB models. ECOM is abbreviated as "E". Top: BDS2 MEO satellites. Bottom: BDS2 IGSO satellites. Left column: 1-day orbit arcs. Middle column: 3-day orbit arcs. Right column: 5-day orbit arcs.

the ephemerides fit the observations at certain epochs (e.g., SLR residuals, satellite clock corrections). Note that the ephemerides related to the so-called long-arcs in the COM solution and in this study are only extracted for the middle day of the respective arc. "Relative" validation methods such as orbit misclosures or long-arc fits characterize, how well independently computed adjacent orbit arcs fit together (i. e., orbit repeatability or consistency). Such methods thus validate the quality of the underlying physical model. A good physical representation can also be assumed if no stochastic parameters have to be set up and if long-arc orbits are of good quality.

The IQR of the SLR residuals related to the MEO satellite SVN C012 in Tabs. 3 and 4 indicates that the ECOM-TB SRP models moderately improve the POD during ON-periods compared to ECOM2. However, the SLR residuals are still larger than during time periods with YS. The solution based on the full SRP model including pulses (ECOM-TBP) performs best, i. e., pulses help to reduce the SLR residuals. With this model the long-arc solution gives slightly better results than the 1-day solution.

The RMS of the linear clock fit grows during ON-periods, as well. The scatter is relatively large, but the different SRP models are more or less on comparable levels for short-arc solutions. Long-arc solutions not using pulses are slightly degraded (ECOM2 more so than ECOM-TB and ECOM-TBM), while solutions with pulses are similar-when compared to the corresponding short-arc solutions (i.e., pulses are contributing to the quality of the MEO long-arc solutions). Note, that the clock statistics of SVN C012 are listed separately in Tab. 4, because its clock is better than that of the other BDS2 MEO satellites.

The orbit repeatability of BDS2 MEO long-arc solutions is significantly better than that of short-arc solutions during YS-periods. During ON-periods the repeatability of the short-arc solutions degrades slightly-when compared to YSperiods. This is independent from the SRP model. The performance of the long-arc solutions, however, shows a stronger 
Table 4: Validation statistics of COM solutions based on different ECOM-TB versions in the time intervals I1 and I2. (IQR stands for inter quartile range; BI for BDS2 IGSO; BM for BDS2 MEO; Q1 for QZS-1; 1 d for 1-day arc; 3 d for 3-day arc)

\begin{tabular}{|c|c|c|c|c|c|c|c|c|}
\hline \multirow[t]{2}{*}{$\begin{array}{l}\text { Sat. } \\
\text {-typ }\end{array}$} & \multicolumn{2}{|c|}{$\begin{array}{c}\text { ECOM-TB } \\
\text { ON }\end{array}$} & \multicolumn{2}{|c|}{$\begin{array}{c}\text { ECOM-TBP } \\
\text { ON }\end{array}$} & \multicolumn{2}{|c|}{$\begin{array}{c}\text { ECOM-TBM } \\
\text { ON }\end{array}$} & \multicolumn{2}{|c|}{$\begin{array}{c}\text { ECOM-TBMP } \\
\text { ON }\end{array}$} \\
\hline & $1 \mathrm{~d}$ & $3 \mathbf{d}$ & $1 \mathrm{~d}$ & $3 \mathbf{d}$ & $1 \mathrm{~d}$ & $3 \mathbf{d}$ & $1 \mathrm{~d}$ & $3 \mathbf{d}$ \\
\hline \multicolumn{9}{|c|}{ SLR residuals $[\mathrm{cm}]:$ median $\pm \mathrm{IQR}$} \\
\hline BI & $-21.1 \pm 28.1$ & $-273.5 \pm 301.2$ & $-20.5 \pm 28.3$ & $-38.8 \pm 59.0$ & $n / a$ & $n / a$ & $-3.5 \pm 7.6$ & $-7.0 \pm 12.2$ \\
\hline $\mathrm{BM}$ & $3.4 \pm 16.4$ & $-6.4 \pm 16.1$ & $3.8 \pm 16.2$ & $-4.6 \pm 12.2$ & $n / a$ & $n / a$ & $-0.9 \pm 10.3$ & $-4.6 \pm 15.0$ \\
\hline Q1 & $-4.5 \pm 28.1$ & $-2.4 \pm 15.2$ & $8.4 \pm 21.7$ & $5.6 \pm 15.9$ & $n / a$ & $n / a$ & $n / a$ & $n / a$ \\
\hline \multicolumn{9}{|c|}{ RMS of linear clock fit [ns]: median \pm IQR } \\
\hline $\mathrm{BI}$ & $1.11 \pm 0.96$ & $5.71 \pm 3.80$ & $1.03 \pm 0.71$ & $1.17 \pm 0.79$ & $1.41 \pm 1.14$ & $1.54 \pm 1.08$ & $0.82 \pm 0.88$ & $0.72 \pm 0.92$ \\
\hline $\mathrm{C} 005$ & $1.70 \pm 0.68$ & $5.70 \pm 2.82$ & $1.39 \pm 0.61$ & $1.52 \pm 0.56$ & $1.85 \pm 1.21$ & $2.04 \pm 0.98$ & $1.81 \pm 0.53$ & $1.70 \pm 0.40$ \\
\hline $\mathrm{BM}$ & $0.89 \pm 0.55$ & $0.92 \pm 0.96$ & $0.72 \pm 0.45$ & $0.69 \pm 0.56$ & $0.90 \pm 0.47$ & $1.05 \pm 0.52$ & $0.78 \pm 0.71$ & $0.72 \pm 0.60$ \\
\hline $\mathrm{C} 012$ & $0.60 \pm 0.36$ & $0.54 \pm 0.19$ & $0.58 \pm 0.34$ & $0.48 \pm 0.29$ & $n / a$ & $n / a$ & $0.51 \pm 0.22$ & $0.54 \pm 0.22$ \\
\hline Q1 & $0.54 \pm 0.41$ & $0.35 \pm 0.17$ & $0.37 \pm 0.31$ & $0.29 \pm 0.09$ & $n / a$ & $n / a$ & $n / a$ & $n / a$ \\
\hline \multicolumn{9}{|c|}{$3 \mathrm{~d}$ orbit misclosures $[\mathrm{cm}]:$ median $\pm \mathrm{IQR}$} \\
\hline $\mathrm{BI}$ & $37.9 \pm 30.9$ & $38.1 \pm 54.1$ & $43.5 \pm 29.9$ & $8.3 \pm 8.3$ & $39.0 \pm 20.7$ & $98.5 \pm 73.3$ & $47.5 \pm 24.9$ & $27.1 \pm 32.0$ \\
\hline $\mathrm{BM}$ & $55.5 \pm 29.5$ & $8.0 \pm 8.4$ & $54.8 \pm 35.2$ & $9.8 \pm 8.9$ & $55.1 \pm 51.5$ & $49.9 \pm 88.9$ & $57.0 \pm 35.5$ & $49.2 \pm 35.1$ \\
\hline Q1 & $31.4 \pm 24.6$ & $14.2 \pm 14.1$ & $35.2 \pm 23.4$ & $10.6 \pm 7.7$ & $n / a$ & $n / a$ & $n / a$ & $n / a$ \\
\hline \multicolumn{9}{|c|}{ RMS of 3-day long arc orbit fit $[\mathrm{cm}]:$ median $\pm \mathrm{IQR}$} \\
\hline $\mathrm{BI}$ & $45.1 \pm 19.7$ & $23.1 \pm 27.3$ & $44.4 \pm 18.1$ & $39.4 \pm 22.0$ & $54.0 \pm 19.0$ & $53.6 \pm 35.4$ & $45.8 \pm 23.9$ & $44.0 \pm 24.5$ \\
\hline $\mathrm{BM}$ & $14.1 \pm 7.1$ & $4.0 \pm 10.8$ & $14.4 \pm 13.8$ & $6.8 \pm 9.5$ & $13.9 \pm 10.9$ & $21.9 \pm 6.6$ & $14.7 \pm 9.4$ & $13.1 \pm 7.5$ \\
\hline Q1 & $11.5 \pm 4.6$ & $5.6 \pm 4.4$ & $11.5 \pm 3.9$ & $5.7 \pm 3.7$ & $n / a$ & $n / a$ & $n / a$ & $n / a$ \\
\hline
\end{tabular}

dependency on the SRP model: Long-arc solutions based on ECOM-TB show significantly smaller orbit misclosures than their short-arc counterparts, while the misclosures remain large for ECOM-TBM and ECOM2. However, the ECOM2-P solution with long arcs benefits significantly from its additional pulses resulting in orbit misclosures comparable to those of the ECOM-TB solution during the ON-periods. Concerning the RMS of long-arc fit, the solution using the full ECOM-TB benefits from longer orbit arcs, while the solution based on ECOM-TBM is degraded. The performance of the ECOM2 lies in between the two extremes. Adding pulses improves the longarc fit of the ECOM2-P and ECOM-TBMP long-arc solutions, but degrades the repeatability of the ECOM-TBP solution.

In summary, the best consistency of BDS2 MEO orbits during the ON-mode can be achieved by using the full ECOM-TB or the ECOM2-P in a long-arc solution. Taking the absolute orbit accuracy into account as well, we prefer ECOM-TBP instead. With this model we are able to significantly reduce the IQR of the SLR residuals $(21.0 \rightarrow 12.2 \mathrm{~cm})$, the RMS of the linear clock fit $(1.6 \rightarrow 0.7 \mathrm{~ns})$, the median of the orbit misclosures $(29.2 \rightarrow 9.8 \mathrm{~cm})$, and the RMS of the long-arc orbit fit $(10.1 \rightarrow$ $6.8 \mathrm{~cm}$ ) in a COM-style 3-day long-arc orbit solution of BDS2 MEO satellites during ON-periods (Tab. 4).

The IQR of the SLR residuals of the BDS2 IGSO satellites SVN C008 and C010 is significantly larger under ON, when compared to YS. For longer arcs the residuals are growing even more (to up to several meters), when using the ECOM-TB or ECOM2 models. Adding pulses (ECOM-TBP) considerably reduces the orbit error of the ECOM-TBP solution. A significant offset of $\approx 40 \mathrm{~cm}$ remains, however, in analogy to the T30b1 coefficient of the same SRP model (Fig. 10, bottom, center and right). The SLR residuals are smallest, when using ECOM-TBMP. The RMS of the linear clock fit confirms the results of the SLR validation. The "minimalist" ECOM-TBM, which is not even supported by pulses, still gives better results than the full ECOM-TB that is suffering from "disturbed" higher-order terms.

The orbit repeatability of BDS2 IGSOs is, in general, degraded during the ON-mode-in particular for short-arc solutions and most obviously in the RMS of the long-arc fit. Solutions based on longer arcs show similar misclosures, but improved long-arc fits, if ECOM-TB is used. The 3-day orbits based on ECOM-TBM show a poor long-arc fit and the misclosures are even more degraded than their 1-day counterparts. The addition of pulses can significantly reduce the orbit misclosures, but does not improve the long-arc fit. The "full" ECOM-TBP with pulses shows the best misclosures $(8.3 \mathrm{~cm})$. In summary, by replacing the ECOM2 by ECOM-TBMP we are able to reduce the IQR of the SLR residuals $(20.5 \rightarrow 12.2 \mathrm{~cm})$, the RMS of the linear clock fit $(1.7 \rightarrow 0.7 \mathrm{~ns})$, and the median of the orbit misclosures $(55.9 \rightarrow 27.1 \mathrm{~cm})$ of a COMstyle 3-day long-arc orbit solution of BDS2 IGSO satellites during ON-mode periods. This significant improvement is, however, achieved with a rather unphysical SRP model, which is reflected by an increased RMS of long-arc orbit fit $(23.0 \rightarrow$ $44.0 \mathrm{~cm})$.

Note, that the clock error of SVN C005 is significantly larger than the average of the BDS2 IGSO satellites under YS (Tab. 3). During ON-periods its clock error is reduced and it is less variable (while it increases for other BDS2 satellites) and is then closer to the other BDS2 IGSO spacecraft (see Tab. 4). This unusual behavior cannot be explained, currently. As the orbit validation did not indicate an exceptionally poor orbit quality of SVN C005 under YS, this effect is likely related to the clock itself (e. g., to thermal clock stability). 


\subsection{Discussion of validation results}
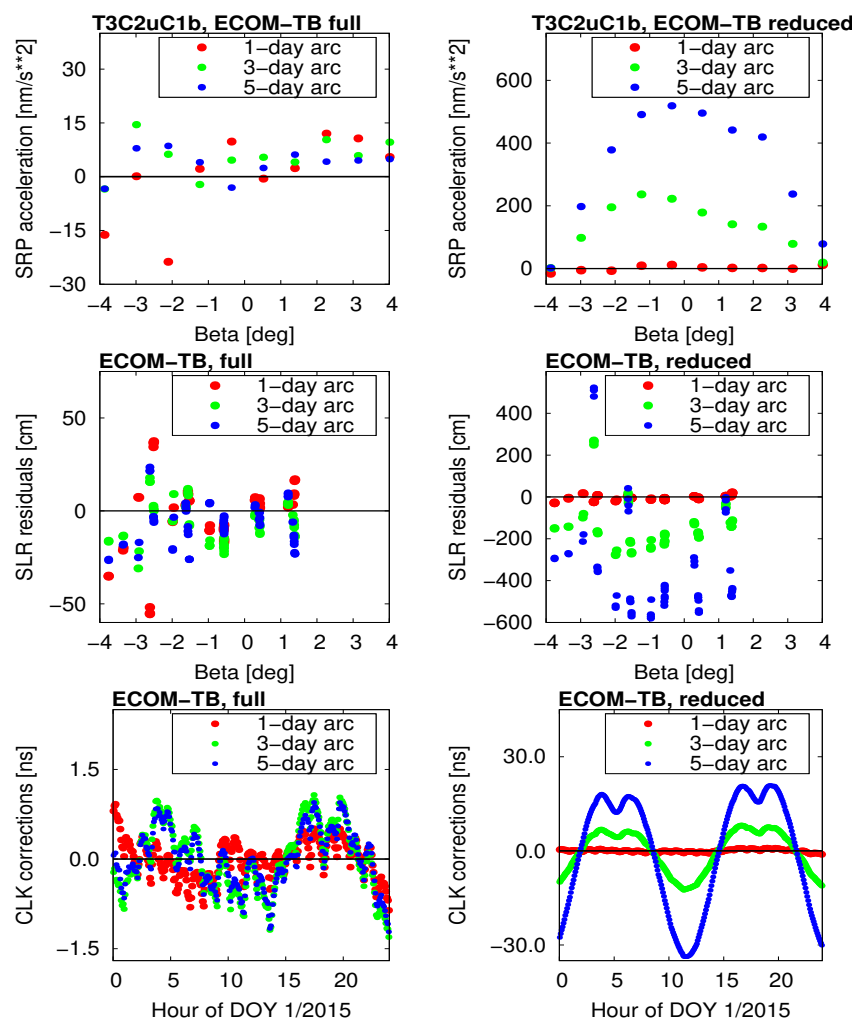

Figure 12: T3C2uC1b SRP coefficient (top), SLR residuals (middle), and clock signal (bottom) of SVN C012 (MEO) for 1-, 3-, and 5-day arcs when using the ECOM-TB SRP model with full (left) or reduced (T3C4uC1b and T3S4uC1b are constrained to zero, right) parameter set.

We showed that the ECOM-TB is an effective SRP model for POD of QZS-1 with long- and short-arcs during ON-mode (Sect. 5.2). It is a bit less effective for POD of BDS2 MEOs, and has problems when applied to BDS2 IGSO long-arcs (Sect. 5.4). Although the ECOM-TB works better for the BDS2 MEOs than for the IGSOs, the following experiment with data of SVN C012 shows that the orbit solution of the BDS2 MEOs is weak, as well:

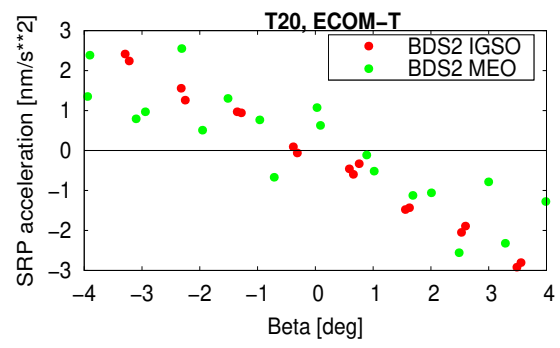

Figure 13: T20 SRP coefficient of ECOM-T as a function of the $\beta$-angle for BDS2 IGSO and MEO satellites.

When constraining the weakly determined (large formal errors, not shown here) coefficients $\mathrm{T} 3 \mathrm{C} 4 \mathrm{uC} 1 \mathrm{~b}$ and $\mathrm{T} 3 \mathrm{~S} 4 \mathrm{uC} 1 \mathrm{~b}$ to zero, other SRP coefficients, such as $\mathrm{T} 3 \mathrm{C} 2 \mathrm{uC} 1 \mathrm{~b}$, are becoming extremely large in the long-arc solutions (Fig. 12, top). The resulting exaggerated signal is ruining the orbit quality (Fig. 12, center) and is clearly visible in the satellite clocks, as well (Fig. 12, bottom). Possibly, the omitted coefficients are absorbing unknown (not necessarily SRP) signal of significant size. Once these parameters are removed, this signal affects some of the remaining parameters. The experiment indicates that we are currently close to the limit when estimating BDS2 long-arc orbits during ON-mode with the full ECOM-TB SRP model-we are slightly below the limit with the MEOs and slightly above with the IGSOs.

Why are long-arc solutions more affected than short-arc solutions? Long-arc solutions are more sensitive to orbit modelling deficiencies, because they accumulate over a longer time span, while the number of arc-specific parameters remains the same. For the 2015 COM solution Prange et al. (2017) list several modelling deficiencies affecting the POD for QZSS and BDS2, such as transmit and receiver antenna phase center offsets, Earth albedo radiation, transmit antenna thrust, and thermal radiation from the spacecraft body.

Is it possible that the BDS2 satellites are not maintaining the ON-mode as assumed for $|\beta|<4^{\circ}$ (implying that we would apply a wrong attitude and an unsuitable SRP model)? Dilssner et al. (2018) reported that some BDS2 MEO and IGSO satellites ceased to enter ON from 2016 onwards. This is, however, not the case in the intervals I1 and I2 in 2014 and 2015. From Fig. 5 we expect that the T20 coefficient of the ECOM-T SRP model is a function of $\sin 3 \beta$ (which is almost linear for $|\beta|<4^{\circ}$ ) for satellites in the ON-mode. When applying ECOM-T to BDS2 satellites we see that this expectation is clearly met for IGSO satellites and a bit less clearly for MEO spacecraft (Fig. 13). For MEOs the T20 coefficient shows larger variations (which agrees with Fig. 11), but a linear trend exists for them, as well. Under YS, the size of T20 would be zero. We therefore conclude that the BDS2 satellites are indeed moving under ON-as expected.

In Fig. 10 (bottom) the T30C1b coefficient deviates significantly from the expected value for the long-arc solutions of the BDS2 IGSO satellites when the "full" ECOM-TB parameterization is used. For the ECOM-TBM (relying on only two constant SRP parameters) this is not the case. The T30C1b coefficient of ECOM-TB must have been "disturbed" by erroneous signals from the periodic coefficients which are missing in ECOM-TBM. In a similar context Prange et al. (2017) reported that the orbits of certain GLONASS spacecraft were degraded by large amplitudes of the 4pr terms of the ECOM2 in the presence of unknown orbit model deficiencies (see Dach et al. 2019, for an in-depth analysis). CODE reacted by de-activating these coefficients in its orbit solutions (Dach et al. 2016). Obviously, the higher order terms of empirical SRP models are more sensitive to orbit model issues than the constant or low-order terms. We assume that a sampling problem (e.g., related to the tracking network geometry) might contribute to the large amplitudes of the higher order terms in Fig. 12, as well.

Why does the full ECOM-TB work better for BDS2 MEO than for BDS2 IGSO satellites? If our assumption of a sampling problem is correct, problems would be correlated with the tracking network. The IGS MGEX network available for the COM solution has relatively few ground stations tracking BDS2 in Northeast Asia (Fig. 14, left). With this network the number 

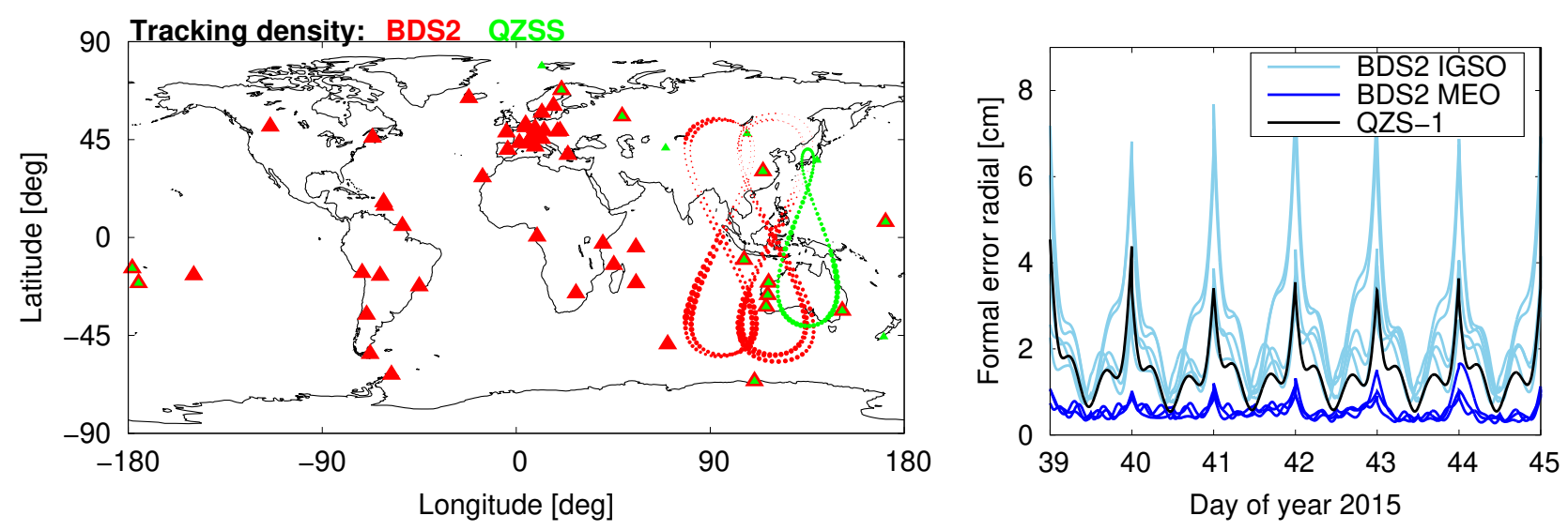

Figure 14: Left: Ground stations (triangles) contributing and ground-tracks of BDS2 (IGSO) and QZS-1 orbits. The width of the lines corresponds with the number of ground stations with view to the satellite at the given location (elevation cutoff: $45^{\circ}$ ). Right: Formal error of radial orbit component (in cm) of QZS-1, BDS2 MEO, and BDS2 IGSO orbits. YS and ECOM2 are used for all satellites.

of stations observing the BDS2 IGSO satellites at high elevations $\left(>45^{\circ}\right)$ varies between 1 and 8 . The lower number corresponds to the Northern hemisphere. For MEO satellites, which still may be observed well in other parts of the world (e.g., over Europe), the difference between minimum and maximum observability is even larger. This deficit may be acceptable for MEOs: The shorter revolution period of the BDS2 MEOs might favor them if the same arc-length is applied for POD of MEOs and IGSOs. Shifting ground tracks, implying changes of the viewing geometry, favor MEOs, as well. For IGSO satellites largely depending on regional tracking, the network configuration might, however, result in a poor and static observation geometry. This hypothesis is actually supported by Fig. 14 (right), showing that the radial orbit error of BDS2 IGSOs is significantly larger than that of BDS2 MEOs and QZS-1.

Missing or wrong meta information about the BDS2 spacecraft may contribute to the differences, as well. Following Han et al. (2011) and Montenbruck et al. (2015), we assume identical spacecraft designs for BDS2 MEO and IGSO satellites in our analysis. The available information is, however, not very detailed. Unexpected design features of the BDS2 IGSO spacecraft might require still another SRP parameterization. The uncertainties concerning the BDS2-related meta data shall be illustrated with the transmit antenna phase center offsets (PCO), which have been estimated by Dilssner et al. (2014), Guo et al. (2017), and Huang et al. (2018). The estimated z-PCOs differ considerably (by up to several meters) - not only from the assumed manufacturer values $(1.1 \mathrm{~m})$ typically used by the MGEX ACs (Montenbruck et al. 2015) and in our analysis, but also among each other. They agree, however, insofar as the PCOs of MEO and IGSO satellites are rather different $(\approx 2 \mathrm{~m}$ vs. $\approx 2.5-4.5 \mathrm{~m}$ ).

Why is the ECOM-TB better for QZS-1 than for BDS2? QZS-1 is well observed along its orbit by ground stations in East-Asia, Southeast-Asia, Australia, and in the Pacific Region (see Fig. 14, left). The data are publicly available via the IGS. QZSS meta-data are known-in particular transmission and receiver antenna offsets. As opposed to BDS2 orbits, the QZSS orbits have substantial eccentricities $(\mathrm{e} \approx 0.075)$. The QZS-1 spacecraft properties have been disclosed (Cabinet Office 2017) to a level of detail allowing a basic judgement whether a certain type of SRP model can work (i.e., whether all important surfaces are sufficiently taken into account by the model). The results presented in Secs. 5.2 and 5.4 prove that the newly developed ECOM-TB is in general applicable for satellites with cubic or elongated bodies in MEO or IGSO orbits. We therefore expect improvements also for BDS2 POD, once the above criteria are met.

Why is the ECOM2-P to some extent competitive with the ECOM-TB models in the POD of BDS2 spacecraft under ON (see Tabs. 3 and 4)? Why is this not the case for POD of QZS-1? ECOM1 and ECOM2 are lacking the coefficients needed to absorb the SRP acceleration component occurring along the $\vec{e}_{T_{2}}$ axis under ON (Sec. 4). While QZS-1 applies the ON-mode for $|\beta|<20^{\circ}$, the BDS2 MEO and IGSO satellites apply ON attitude for $|\beta|<4^{\circ}$. Hence, the maximum deviation from the nominal YS is much smaller for BDS2 than for QZS-1 and the modelling deficiency of the ECOM1 and ECOM2 is less significant. Moreover, we have shown that our BDS2 orbit analysis is affected by considerable modelling deficiencies superimposing the SRP model errors. The pseudo-stochastic orbit parameters of the ECOM2-P, ECOM-TBP, and ECOM-TBMP solutions are able to absorb error signal of different sources, thus mitigating the effect of the SRP model.

\section{Comparison with external MGEX orbits}

Table 5 compares the original COM orbits (COMo, based on ECOM2) and a COM test solution (COMn) based on the newly developed ECOM versions (ECOM-TBMP for BDS2 IGSO, ECOM-TBP for BDS2 MEO, ECOM-TB for QZS-1) with orbits provided by other ACs contributing to the IGS MGEX (Montenbruck et al. 2017b). Like in the previous sections all ECOM models are used without an a priori SRP model.

The COMo solution has the largest IQR of the SLR residuals for all listed satellite types and the largest median orbit misclosures for the BDS2 spacecraft. The best QZS-1 orbits are 
provided by QZF, probably because they use a dedicated a priori SRP model (Ikari 2018). The smallest IQR of SLR residuals for BDS2 orbits and the smallest misclosures for BDS2 MEOs results for the WUM solution. The BDS2 IGSO misclosures, however, are better for the GBM solution.

With the empirical SRP models of the ECOM-TB family applied, the POD results of the COMn solution are competitive: For COMn the IQR of the SLR residuals for QZS-1 is only marginally larger than for the QZF solution (15.2 vs. $14.3 \mathrm{~cm})$. The orbit misclosures are significantly reduced $(42.4$ $\rightarrow 14.2 \mathrm{~cm}$ ) and are on rank 2, behind the QZF solution. The good agreement between the COMn and QZF solutions is confirmed by orbit comparisons done in the frame of the IGS MGEX (IGS-MGEX 2019). An SLR validation performed by Steigenberger (2018) indicates that ECOM-TB is comparable in performance to the ray-tracing model developed by Darugna et al. (2018).

The COMn BDS2 MEO solution is also on the second rank (behind WUM) regarding IQR of SLR residuals and shows the best orbit misclosures. The COMn BDS2 IGSO orbits are of the same quality as the WUM solution concerning the IQR of the SLR residuals, however, at the price of a significant SLR offset. The orbit misclosures of this solution are on a similar level as those from GBM. In summary, the ECOM-TB family of SRP models allows the generation of BDS2 and QZS-1 orbits, which are competitive with the best solutions of other MGEX ACs for satellites moving in the $\mathrm{ON}$-mode.

Table 5: Median (M) and IQR of SLR residuals (SLR) and orbit misclosures (OMC) of MGEX orbits from different ACs during ON-seasons within the time interval DOY 355/2014-300/2015. Unit: cm. COMo and COMn: original and new (based on ECOM-TB(M)) COM orbits. GBM: GFZ Potsdam. QZF: QZSS operations center. TUM: TU München. WUM: Wuhan University.

\begin{tabular}{llrr} 
GNSS & AC & $\begin{array}{r}\text { SLR } \\
\text { M } \pm \text { IQR }\end{array}$ & $\begin{array}{r}\text { OMC } \\
\text { M } \pm \mathbf{I Q R}\end{array}$ \\
\hline BDS2 IGSO & COMo & $1.5 \pm 20.5$ & $55.9 \pm 49.0$ \\
& COMn & $-7.0 \pm 12.2$ & $27.1 \pm 32.0$ \\
& GBM & $3.3 \pm 13.8$ & $30.9 \pm 28.7$ \\
BDS2 MEO & WUM & $0.3 \pm 12.4$ & $38.9 \pm 34.5$ \\
& COMo & $-3.8 \pm 21.0$ & $29.2 \pm 58.7$ \\
& COMn & $-4.6 \pm 12.2$ & $9.8 \pm 8.9$ \\
& GBM & $3.1 \pm 16.3$ & $28.1 \pm 48.3$ \\
& WUM & $0.0 \pm 8.7$ & $16.4 \pm 20.7$ \\
& COMo & $9.4 \pm 62.0$ & $42.4 \pm 59.9$ \\
& COMn & $-2.4 \pm 15.2$ & $14.2 \pm 14.1$ \\
& GBM & $33.3 \pm 37.7$ & $108.2 \pm 101.0$ \\
& QZF & $-11.4 \pm 14.3$ & $6.5 \pm 3.7$ \\
& TUM & $18.7 \pm 49.5$ & $61.0 \pm 59.3$ \\
& WUM & $30.9 \pm 46.6$ & $113.2 \pm 91.2$ \\
\hline
\end{tabular}

\section{Conclusions}

In Sect. 2 the terminator (TERM) system was defined as the reference frame for an empirical SRP model, which is well suited for satellites moving in the ON-mode. As opposed to the classical ECOM decomposition the TERM system is not co-rotating with the satellite, but with the Sun elevation angle $\beta$ over the orbital plane.
Based on theoretical insight and on the comparison of SRP accelerations acting on satellites moving under the YS- and ON-mode (Sect. 3), respectively, we defined the main components of the SRP acceleration (Sect. 4.1), caused by the illumination of the solar panels. As opposed to the YS mode, the SRP model for the solar panels (in general the largest SRP component) depends on the $\beta$ angle for ON-motion. In the TERM system the SRP accelerations due to the solar panels is absorbed by only two coefficients. Based on a simulation study we add terms accounting for the more complex SRP due to the satellite body rotating in the TERM system (Sect. 4.2). The coefficients of the resulting ECOM-T SRP model depend on the $\beta$-angle. As $\beta$ is usually changing in time due to the precession of the orbital plane, the nine coefficients of ECOM-T are a function of time, as well. The final result is the model ECOM-TB with constituents defined as functions of the satellite's argument of latitude $\Delta u$ w.r.t. the Sun and the $\beta$-angle. By taking the $\beta$ angle into account, the coefficients of ECOM-TB are almost constant in time-definitely an advantage for POD using long arcs.

When applied to QZS-1, the estimated coefficents of ECOM-TB agree well with the expectations raised by the simulation (Sect. 5.1). Orbit and clock validations confirm that the ECOM-TB significantly improves the satellite orbits and clock corrections of QZS-1 during ON-periods, when compared to ECOM2 (Sect. 5.2): The IQR of SLR residuals and the orbit misclosures improve by factors of 4 and 3 , respectively. Comparisons to other MGEX solutions (Sect. 6) indicate that our QZS-1 orbits are competitive, demonstrating that the ECOM-TB is well suited for SRP modelling of (IGSO) satellites moving under ON.

A problem remains with BDS2: Firstly, there is a lack of meta data (e. g., transmission and receiver antenna calibrations, satellite mass, optical properties and sizes of the main satellite surfaces). Secondly, the tracking data available to the IGS has gaps for BDS - in particular in East Asia. The higher order terms of the empirical SRP models are sensitive to both aspects. For MEOs the tracking geometry is variable and the poor observation coverage over Asia is partly compensated by a better coverage in other parts of the world. For IGSO satellites this compensation is not possible. The degraded observation geometry of BDS IGSOs is reflected by elevated formal orbit errors (Sect. 5.5) compared to QZS-1 and BDS2 MEOs. As a result the orbits and satellite clock corrections under ONmode improve only moderately compared to the ECOM2 for BDS2 MEOs and may even get worse for BDS2 IGSO satellites (Sect. 5.4). In order to achieve a reasonable accuracy of BDS2 IGSO ephemerides during ON-periods we therefore define a modified version of the ECOM-TB, called ECOM-TBMP, where the periodic terms are replaced by pseudo-stochastic pulses in the radial, along-track, and cross-track directions. With this modified model the ephemerides and satellite clock corrections of BDS2 IGSOs can be improved by a factor of about two compared to ECOM2. The long-arc fit, however, becomes worse-indicating that this stochastic model is not well suited for orbit prediction. Pulses may also absorb remaining modelling deficits of the original ECOM-TB, resulting in the 
ECOM-TBP. The orbits and clocks of BDS2 MEOs can be improved by a factor of about two when replacing the ECOM 2 by the ECOM-TBP.

We expect to see an improved performance of the full ECOM-TB also for BDS2, once better background models and observation coverage will be available. In the current situation described above we decided to activate different versions of the ECOM-TB model family (ECOM-TB for QZS-1, ECOM-TBP for BDS2 MEOs, ECOM-TBMP for BDS2 IGSOs) in the COM solution in summer 2018-replacing ECOM2 during periods with $\mathrm{ON}$-attitude. The data-driven algorithm now implemented in the COM routine, detected that the $\beta$-angle at which QZS-1 performs its $\mathrm{YS} \rightarrow \mathrm{ON}$ and $\mathrm{ON} \rightarrow \mathrm{YS}$ switches has changed from $\pm 20^{\circ}$ to $\pm 17^{\circ}$ since early 2017 .

We did not use a priori SRP models in our analysis. The models of the ECOM-TB family (like all ECOM models) might, however, be combined with an a priori model. In particular the ECOM-TBM version appears to be well suited for this purpose, as it models the SRP due to the solar panels with only two zeroorder coefficients, while the a priori model would have the focus on SRP acting on the spacecraft body. The combined analysis of box-wing models and different ECOM versions demands further research.

\section{Acknowledgement}

We thank all institutions providing and distributing raw data of MGEX stations and the ILRS for providing SLR measurements to a variety of GNSS satellites.

\section{References}

Arnold, D., Meindl, M., Beutler, G., Dach, R., Schaer, S., Lutz, S., Prange, L., Sośnica, K., Mervart, L., Jäggi, A., 2015. CODE's New Solar Radiation Pressure Model for GNSS Orbit Determination. Journal of Geodesy 89, 775-791. doi:10.1007/s00190-015-0814-4.

Beutler, G., 2005. Methods of Celestial Mechanics. Springer-Verlag, Berlin, Heidelberg, New York.

Beutler, G., Brockmann, E., Gurtner, W., Hugentobler, U., Mervart, L., Rothacher, M., Verdun, A., 1994. Extended Orbit Modeling Techniques at the CODE Processing Center of the International GPS Service for Geodynamics (IGS): Theory and Initial Results. Manuscripta Geodaetica 19, $367-386$.

Cabinet Office, 2017. Government of Japan, QZSS Satellite Information. URL: http://qzss.go.jp/en/technical/qzssinfo/. Last access: 31 Jan. 2019.

Colombo, O.L., 1989. The Dynamics of Global Positioning Orbits and the Determination of Precise Ephemerides. Journal of Geophysical Research 94, 9167-9182.

Dach, R., Schaer, S., Arnold, D., Orliac, E., Prange, L., Sušnik, A., Villiger, A., Maier, A., Mervart, L., Jäggi, A., Beutler, G., Brockmann, E., Ineichen, D., Lutz, S., Wiget, A., Rülke, A., Thaller, D., Habrich, H., Söhne, W., Ihde, J., Hugentobler, U., 2016. CODE Analysis Center Technical Report 2015, in: Jean, Y., Dach, R. (Eds.), International GNSS Service: Technical Report 2015, IGS Central Bureau. pp. 25-44. doi:10.7892/boris.80307.

Dach, R., Sušnik, A., Grahsl, A., Villiger, A., Schaer, S., Arnold, D., Prange, L., Jäggi, A., 2019. Improving GLONASS Orbit Quality by Re-estimating Satellite Antenna Offsets. Advances in Space Research 63, 3835-3847. doi:10.1016/j.asr.2019.02.031.

Darugna, F., Steigenberger, P., Montenbruck, O., Casotto, S., 2018. Ray-tracing solar radiation pressure modeling for QZS-1. Advances in Space Research 62, 935-943. doi:10.1016/j.asr.2018.05.036.
Dilssner, F., Läufer, G., Springer, T., Schönemann, E., Enderle, W., 2018. The BeiDou Attitude Model for Continuous Yawing MEO and IGSO Spacecraft. Presentation, EGU General Assembly 2018, Vienna, Austria.

Dilssner, F., Springer, T., Schönemann, E., Enderle, W., 2014. Estimation of Satellite Antenna Phase Center Corrections for BeiDou. Poster, IGS Workshop 2014, Pasadena, USA.

Fliegel, H.F., Gallini, T.E., Swift, E.R., 1992. Global Positioning System radiation force model for geodetic applications. Geophysical Research Letters 97, 559-568. doi:10.1029/91JB02564.

Guo, J., Chen, G., Zhao, Q., Liu, J., Liu, X., 2017. Comparison of solar radiation pressure models for BDS IGSO and MEO satellites with emphasis on improving orbit quality. GPS Solutions 21, 511-522. doi:10.1007/s10291-016-0540-2.

Han, C., Yang, Y., Cai, Z., 2011. BeiDou Navigation Satellite System and its time scales. Metrologia 48, 213-S218. doi:10.1088/0026-1394/48/4/S13.

Huang, G., Yan, X., Zhang, Q., 2018. Estimation of antenna phase center offset for BDS IGSO and MEO satellites. Presentation, IGS Workshop 2018, Wuhan, China.

IGS-MGEX, 2019. International GNSS Service, MGEX Product Analysis. URL: http: //mgex .igs . org/analysis/. Last access: 31 Jan. 2019.

Ikari, S., 2018. A priori solar radiation pressure model used for the QZF solution. Personal communication, October 2018.

Johnston, G., Riddell, A., Hausler, G., 2017. The International GNSS Service. In: Springer Handbook of Global Navigation Satellite Systems. doi:10.1007/978-3-319-42928-1.

Lou, Y., Liu, Y., Shi, C., Yao, X., Zheng, F., 2014. Precise orbit determination of BeiDou constellation based on BETS and MGEX network. Sci. Rep. 4. doi:10.1038/srep04692.

Lutz, S., Meindl, M., Steigenberger, P., Beutler, G., Sośnica, K., Schaer, S., Dach, R., Arnold, D., Thaller, D., Jäggi, A., 2016. Impact of the arc length on GNSS analysis results. Journal of Geodesy 90, 365-378. doi:10.1007/s00190-015-0878-1.

Milani, A., Nobili, A., Farinella, P., 1987. Non-gravitational Perturbations and Satellite Geodesy. Adam Hilger Ltd., Bristol, UK.

Montenbruck, O., Schmid, R., Mercier, F., Steigenberger, P., Noll, C., Fatkulin, R., Kogure, S., Ganeshan, A., 2015. GNSS satellite geometry and attitude models. Advances in Space Research 56, 1015-1029. doi:10.1016/j.asr.2015.06.019.

Montenbruck, O., Steigenberger, P., Darugna, F., 2017a. Semi-analytical solar radiation pressure modeling for QZS-1 orbit-normal and yawsteering attitude. Advances in Space Research 59, 2088-2100. doi:10.1016/j.asr.2017.01.036

Montenbruck, O., Steigenberger, P., Prange, L., Deng, Z., Zhao, Q., Perosanz, F., Romero, I., Noll, C., Stürze, A., Weber, G., Schmid, R., MacLeod, K., Schaer, S., 2017b. The Multi-GNSS Experiment (MGEX) of the International GNSS Service (IGS) - Achievements, prospects and challenges. Advances in Space Research 59, 1671-1697. doi:10.1016/j.asr.2017.01.011. article.

Pearlman, M.R., Degnan, J.J., Bosworth, J.M., 2002. The International Laser Ranging Service. Advances in Space Research 30, 135-143. doi:10.1016/S0273-1177(02)00277-6.

Prange, L., Orliac, E., Dach, R., Arnold, D., Beutler, G., Schaer, S., Jäggi, A., 2017. CODE's five-system orbit and clock solution-the challenges of multi-GNSS data analysis. Journal of Geodesy 91, 345-360. doi:10.1007/s00190-016-0968-8.

Rodriguez-Solano, C., Hugentobler, U., Steigenberger, P., 2012. Adjustable box-wing model for solar radiation pressure impacting GPS satellites. Advances in Space Research 49, 1113 - 1128. doi:10.1016/j.asr.2012.01.016.

Springer, T.A., 1999. Modeling and Validating Orbits and Clocks Using the Global Positioning System. Ph.D. thesis. Astronomical Institute, University of Berne. Bern, Switzerland.

Steigenberger, P., 2018. SLR validation of QZS-1 orbits based on different solar radiation pressure models. Personal communication, March 2018.

Zhao, Q., Chen, G., Guo, J., Liu, J., Liu, X., 2018. An a priori solar radiation pressure model for the QZSS Michibiki satellite. Journal of Geodesy 92, 109-121. doi:10.1007/s00190-017-1048-4.

Zhao, Q., Guo, J., Li, M., Qu, L., Hu, Z., Shi, C., Liu, J., 2013. Initial results of precise orbit and clock determination for COMPASS navigation satellite system. JG 87, 475-486. doi:10.1007/s00190-013-0622-7. 